\title{
Combined Experimental and Computational Investigation of 2-(2-Hydroxyphenylimino) Phenolic Derivatives: Synthesis, Molecular Structure and NLO Studies
}

\author{
V. Duraisamy Nadhiya1, Rathinam Kumaresan ${ }^{2}$ \\ ${ }^{1}$ Research Department of Physics, Kongunadu Arts and Science College (Autonomous), Coimbatore, India \\ ${ }^{2}$ Research Department of Physics, Government Arts College (Autonomous), Coimbatore, India \\ Email: drkumaresanrathinam@gmail.com
}

How to cite this paper: Nadhiya, V.D. and Kumaresan, R. (2017) Combined Experimental and Computational Investigation of 2-(2-Hydroxyphenylimino) Phenolic Derivatives: Synthesis, Molecular Structure and NLO Studies. International Journal of Organic Chemistry, 7, 185-217.

https://doi.org/10.4236/ijoc.2017.72015

Received: May 4, 2017

Accepted: June 25, 2017

Published: June 28, 2017

Copyright $\odot 2017$ by authors and Scientific Research Publishing Inc. This work is licensed under the Creative Commons Attribution International License (CC BY 4.0).

http://creativecommons.org/licenses/by/4.0/

\begin{abstract}
A series of substituted 2-(2-hydroxyphenylimino) phenolic (salen) derivatives (1-4) have been synthesized and their structures of obtained compound were characterized by analytical, FT-IR, UV-Vis and ${ }^{13} \mathrm{C}\left\{{ }^{1} \mathrm{H}\right\}$-NMR experimentally. The geometry structure optimization, frequencies (IR), NMR, electronic character, frontier molecular orbital (HOMO-LUMO) and first static hyperpolarizability $\left(\beta_{\text {tot }}\right)$ studies of reported compounds were calculated using DFT with B3LYP/6-311G(d,p) level of theory. The calculated HOMO and LUMO energies showed that charge transfer occurs within the molecule and from the MEP, the molecular stability and bond strength have been explained. In addition to that influence of energy gap $\left(\Delta \mathrm{E}_{\text {gap }}\right)$ between the HOMO-LUMO orbitals on the first static hyperpolarizability $\left(\beta_{\text {tot }}\right)$, we calculated the $\Delta \mathrm{E}_{\text {gap }}$ for all the salen compounds. These results reveals that the smaller HOMO-LUMO $\Delta \mathrm{E}_{\text {gap }}$ is, larger the $\beta_{\text {tot }}$ is.
\end{abstract}

\section{Keywords}

Salen, PES, HOMO-LUMO, MEP, $\Delta \mathrm{E}_{\text {gap }}$, Hyperpolarizability

\section{Introduction}

Schiff bases are important class of organic compounds have long attracted attention, owing to their remarkable biological and pharmacological properties, such as antibacterial, antiviral, antineoplastic and antimalarial activities. The functional applications of the azomethine group of Schiff base derivatives enable their use in numerous fields, they have 
$>$ nucleophilic imine group,

$>$ an imine carbon that has both electrophilic and nucleophilic character,

$>$ configurations isomerism from the presence of the $\mathrm{C}=\mathrm{N}$ double bond.

These structural features of the Schiff base compounds give its physical and chemical properties [1] [2] [3]. Schiff bases constitute some of the most valuable groups of biomolecules. First reported in 1864 by Hugo Schiff, these compounds gained notoriety due to the ease way of preparation from commercially available inexpensive aldehydes/ketones and primary amines. The azomethine linkage $(>\mathrm{C}=\mathrm{N}-)$ allows rapid access to vast libraries of structurally diverse molecular hybrids with interesting biological properties, including antifungal, antibacterial, antimalarial, anti-inflammatory, antiviral, antioxidant, pesticidal and in-vitrol in-vivo inhibitory effects against experimental tumor cells. The electrophilic carbon and nucleophilic nitrogen in $(>\mathrm{C}=\mathrm{N}-)$ core confer to Schiff bases the possibility to interact with several nucleophilic and electrophilic biological species, which can lead to enzymes inhibition or DNA replication impairment. Then, Schiff bases are promising as lead compounds for the rational design of novel cytotoxic and cytostatic small molecules with a mechanism of action that may differ from that of clinically approved anticancer agents [3] [4] [5] [6].

In addition, Schiff base compounds high potential applications in non-linear optical (NLO), optical communication, optical signal processing and transmission, optical data acquisition and storage, optical computing, and especially optical limiting effects utilized in the protection of optical sensors and human eyes from high-intensity laser beams [7] [8] [9] [10]. In the present work, we have synthesized certain salen based Schiff base compounds, 2-(2-hydroxyphenylimino) phenolic derivatives viz. salcylaldehyde (SA), o-hydroxyacetophenone (AA), $o$-vanillin (VA) and 2-hydroxy-1-naphthaldehyde (NA), with $o$-aminophenol [11] [12] [13] [14] and characterized by FT-IR, UV-Vis, ${ }^{13} \mathrm{C}\left\{{ }^{1} \mathrm{H}\right\}-\mathrm{NMR}$ techniques, Cyclic Voltammetry studies. Moreover, theoretical studies were carried out on the molecular structure using density functional methods (B3LYP) invoking 6-311G(d,p) basis set. The energy of the highest occupied molecular (HOMO) orbital and lowest unoccupied (LUMO) molecular orbital have been predicted.

\section{Experimental}

\section{Materials and Methods}

All the chemicals and solvents used were purified and dried by standard methods. FT-IR spectra were recorded as $\mathrm{KBr}$ pellets with a PerkinElmer FT-IR spectrometer in $4000-400 \mathrm{~cm}^{-1}$ range. Microanalyses were carried out with a Vario El AMX-400 elemental analyzer at STIC, Cochin University of Science and Technology, Kerala, India. Electronic spectra were recorded in $\mathrm{CH}_{3} \mathrm{CN}$ as solvent with an Ocean optics spectrophotometer USB 4000. NMR spectra were also recorded on Bruker AVANCE III $500 \mathrm{MHz}$ (AV 500) spectrometer; chemical shifts are expressed in ppm ( $\delta$ units) relative to TMS signal as internal reference in DMSO.d $\mathrm{d}_{6}$. 
The Schiff base compounds (Scheme 1) were prepared by the reported literature procedure [11] [12] [13] [14] with modification of the substitutions and the purity of the Schiff bases were checked by TLC. Melting points were recorded with a Inlab, India micro heating table and were uncorrected.

To an ethanolic solution of salcylaldehyde (SA), o-hydroxyacetophenone (AA), o-vanillin (VA) and 2-hydroxy-1-naphthaldehyde (NA) $\left(2 \mathrm{~cm}^{3}, 20 \mathrm{mmol}\right)$, with o-aminophenol $\left(2 \mathrm{~cm}^{3}, 20 \mathrm{mmol}\right)$ were added with stirring. The mixture was then refluxed for $4-6$ hours with the controlled conditions about $140^{\circ} \mathrm{C}$ $180^{\circ} \mathrm{C}$. On cooling the solution, a solid compound which separated out was filtered, dried and recrystallized from ethanol/DMSO (75:25). The purity of the ligand was checked by TLC. Yield, $85 \%$, m.p. $143.6^{\circ} \mathrm{C}-245^{\circ} \mathrm{C}$.

All the salen compunds were stable at room temperature, non-hygroscopic and insoluble in water partially soluble in methanol, ethanol and soluble in $\mathrm{CH}_{2} \mathrm{Cl}_{2}, \mathrm{CHCl}_{3}, \mathrm{DMF}$, DMSO, $\mathrm{CH}_{3} \mathrm{CN}$, etc.

\section{Computational Methods}

All the computational studies have been carried out with the GAUSSIAN 03W program package [15]. Density functional theory (DFT) method has been applied because of its excellent compromise between computational time and description of electronic correlation and qualitative structure-activity relationships (QSAR) studies [16]. B3LYP, a hybrid functional of the DFT method, which consists of the Becke's three parameters exact exchange functional B3 [17] combined with the non-local gradient corrected correlation functional of Lee-YangParr (LYP) [18] has been used. The standard triple split valence basis set 6-311G [19] with a set of $d, p$ polarization functions on heavy atoms and hydrogen atoms are used throughout the computational process.

A fully relaxed potential energy scan was carried out against the dihedral angle C2-C1-C7-N1 (Scheme 2) at B3LYP/6-311G(d,p) level, the minimum energy conformations from the energy scan, a further geometry optimization was performed at the same level of theory. Vibrational frequencies of the optimized structures were computed using the same level of theory and thermodynamic

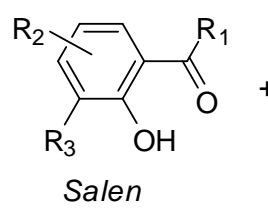

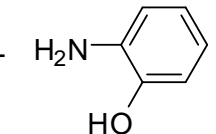

o-aminophenol

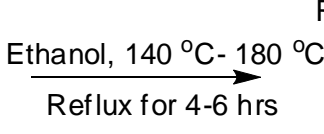

Reflux for 4-6 hrs

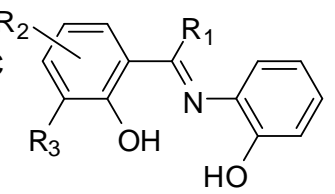

Salen Schiff bases

\begin{tabular}{ccccc}
\hline S.No. & $\mathbf{R}_{\mathbf{1}}$ & $\mathbf{R}_{2}$ & $\mathbf{R}_{\mathbf{3}}$ & Abbreviation \\
\hline 1. & $\mathrm{H}$ & $\mathrm{H}$ & $\mathrm{H}$ & SA \\
2. & $\mathrm{CH}_{3}$ & $\mathrm{H}$ & $\mathrm{H}$ & $\mathbf{A A}$ \\
3. & $\mathrm{H}$ & $\mathrm{H}$ & $\mathrm{OCH}_{3}$ & VA \\
4. & $\mathrm{H}$ & $\mathrm{C}_{4} \mathrm{H}_{4}$ & $\mathrm{H}$ & NA \\
\hline
\end{tabular}

Scheme 1. Preparation of Schiff base compounds. 

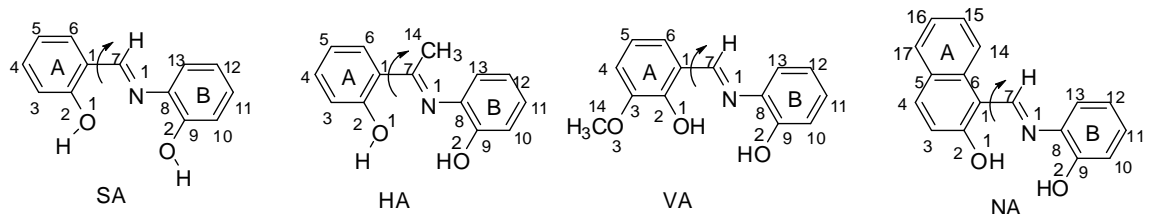

Scheme 2. Potential energy scan (PES) for salen compounds.

corrections [20] [21] were obtained at $298 \mathrm{~K}$ and $1 \mathrm{~atm}$, and added to electronic energies.

Calculated electronic properties such as dipole moment, first static hyper-polarizability HOMO and LUMO energies, MEP, energy gap, electronic affinity $(E A)$, electronegativity $(\chi)$, hardness $(\eta)$, softness $(S)$, electrophilic index $(\omega)$ and ionization potential (IP) have been studied for all the four compounds under consideration. The DFT-based reactivity descriptors were obtained from the Equations (1)-(4) [22] [23] [24] [25] which play an important role in many areas of research.

Electronegativity $(\chi)$

$$
\chi=-\mu=-\left[\frac{I P+E A}{2}\right]
$$

Hardness $(\eta)$

$$
\eta=\left[\frac{I P-E A}{2}\right]
$$

Softness $(S)$

$$
S=\left[\frac{1}{2 \eta}\right]
$$

The experimental polarizability is obtained as an average polarizability, given by $\langle\alpha\rangle=\frac{1}{3}\left(\alpha_{x x}+\alpha_{y y}+\alpha_{z z}\right)$ and the computed average polarizability is also presented.

\section{Results and Discussion}

\subsection{Experimental Results}

\subsubsection{Elemental Analysis and FT-IR Spectral Data}

The elemental analysis data (Table 1) for the all Schiff base compounds are well agreed with the proposed molecular formulae.

The FT-IR spectroscopy is a powerful tool for the assignments of fundamental functional group determinations of organic compounds. In the title compounds frequencies of the functional groups viz., $v_{>\mathrm{C}=\mathrm{N}-}, v_{\mathrm{ph}-\mathrm{OH}}, v_{\mathrm{ph}-\mathrm{C}-\mathrm{O}}, v_{\mathrm{ph}-\mathrm{N}=\mathrm{C}-}$ and $v_{-\mathrm{N}=\mathrm{CH}} / v_{-\mathrm{N}=\mathrm{C}(\mathrm{CH} 3)}$ are of great importance in the infrared spectra. The infrared spectra of all the four Schiff bases (Figure 1) exhibited a strong band in the 1692 - $1642 \mathrm{~cm}^{-1}$ region due to the characteristic of azomethine $v(\mathrm{C}=\mathrm{N})$ group. All the title compounds were displayed a band in the region $3399-3308 \mathrm{~cm}^{-1}$, which could be due to $v(\mathrm{ph}-\mathrm{OH})$. A strong band observed at $1373-1362 \mathrm{~cm}^{-1}$ in 

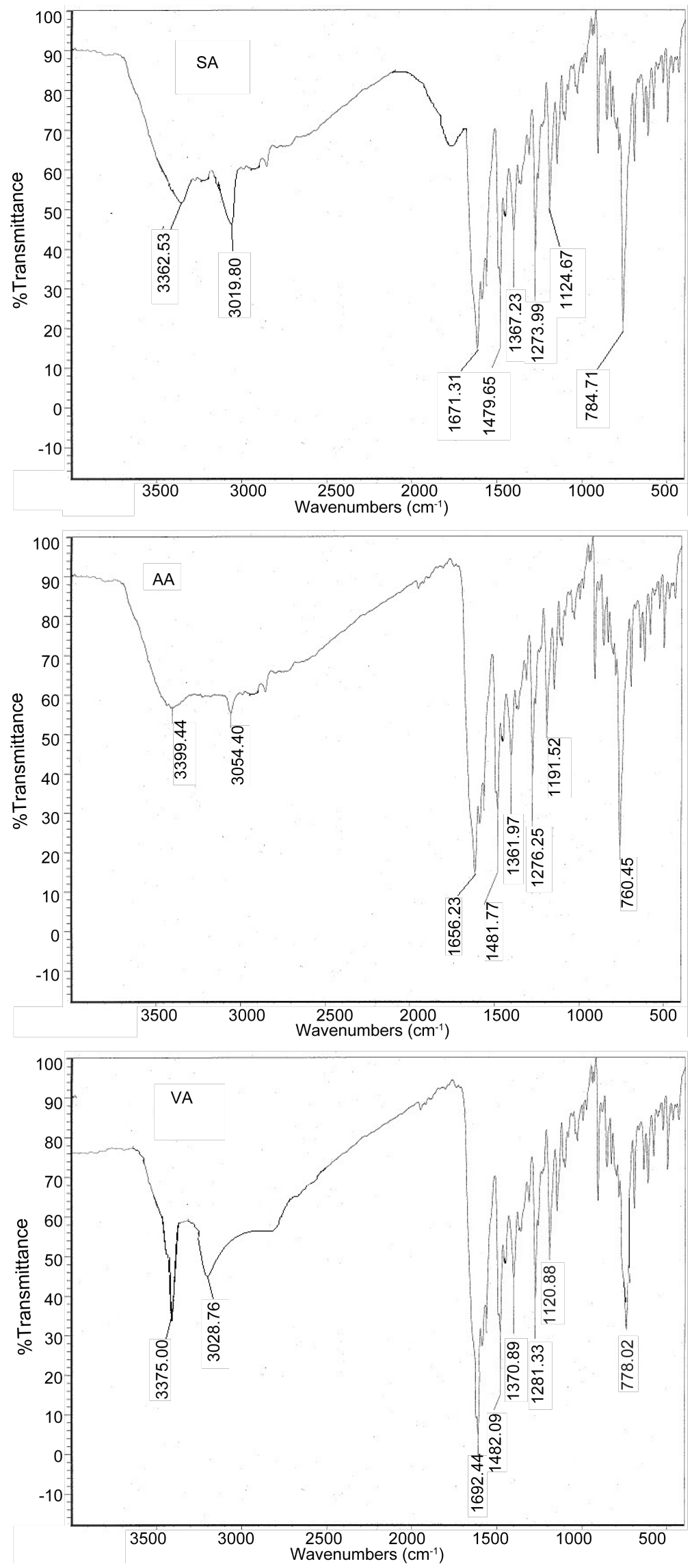


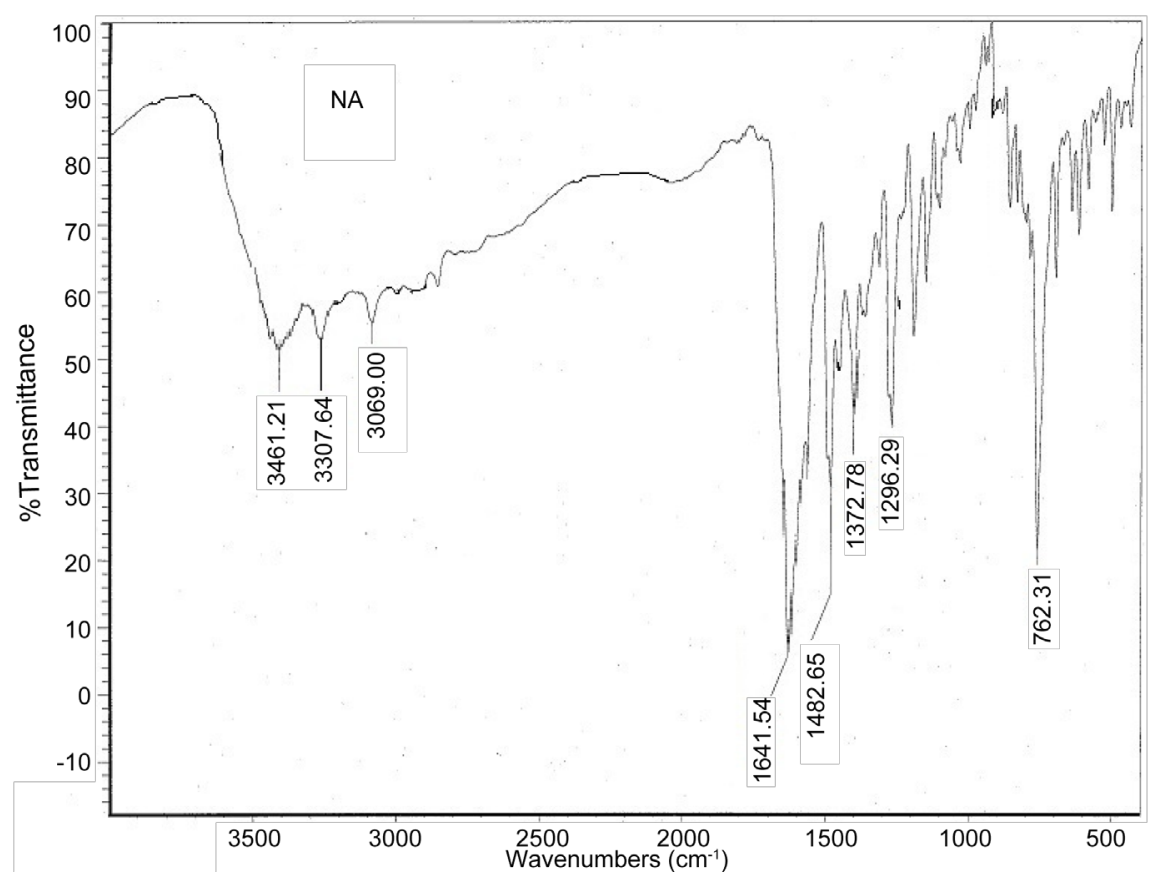

(a)

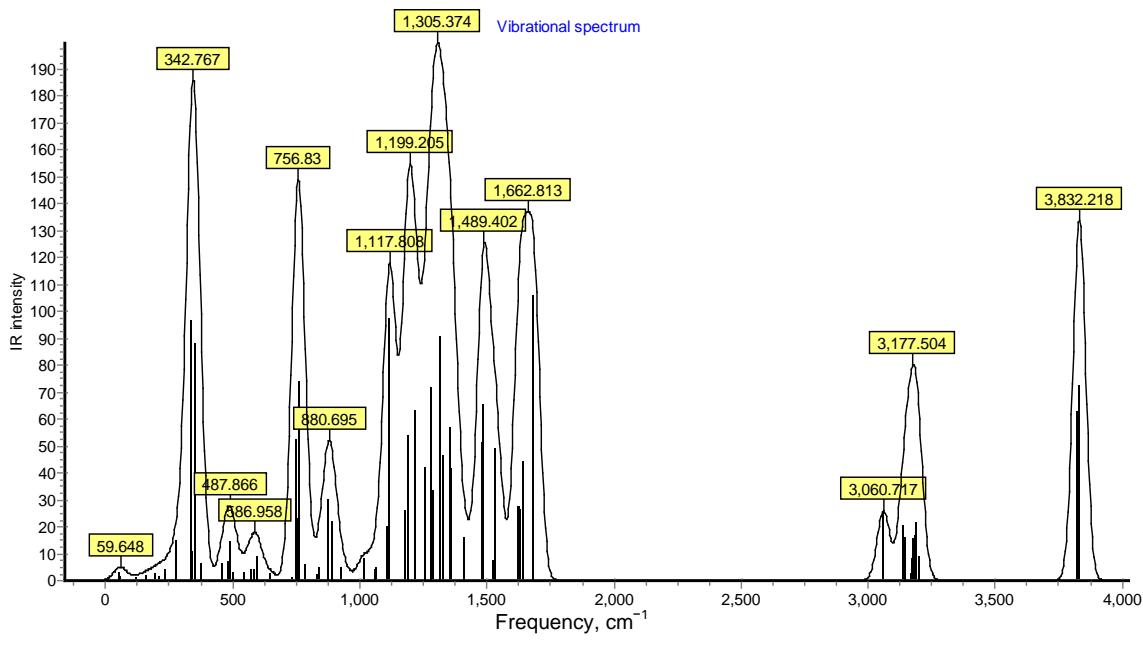

IR SPECTRUM OF SA

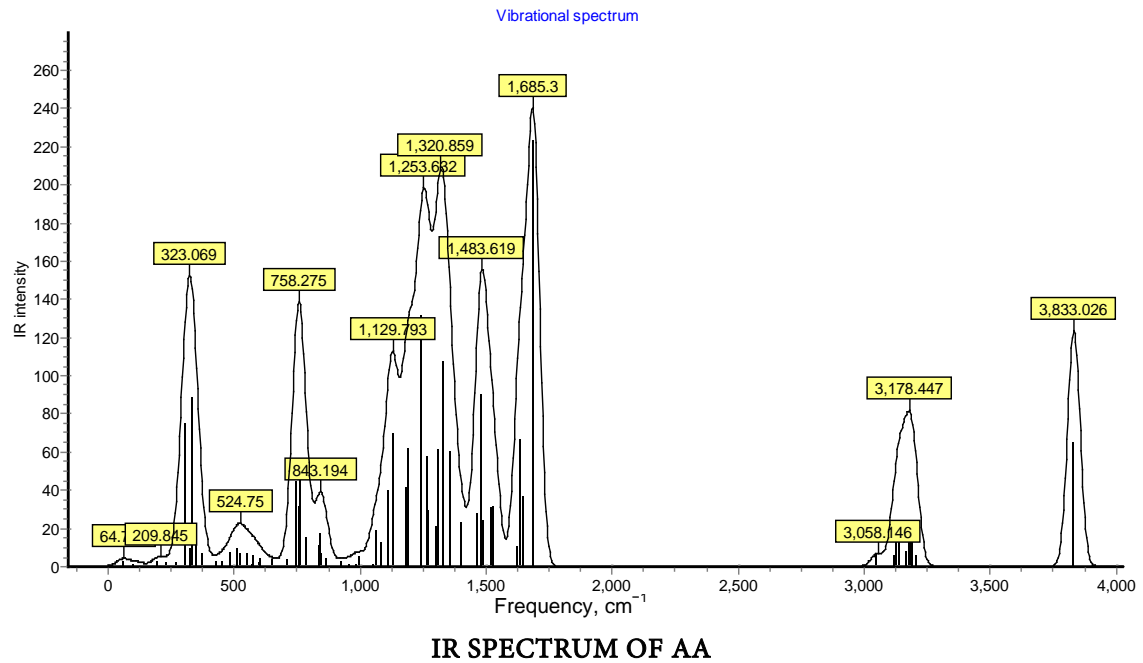




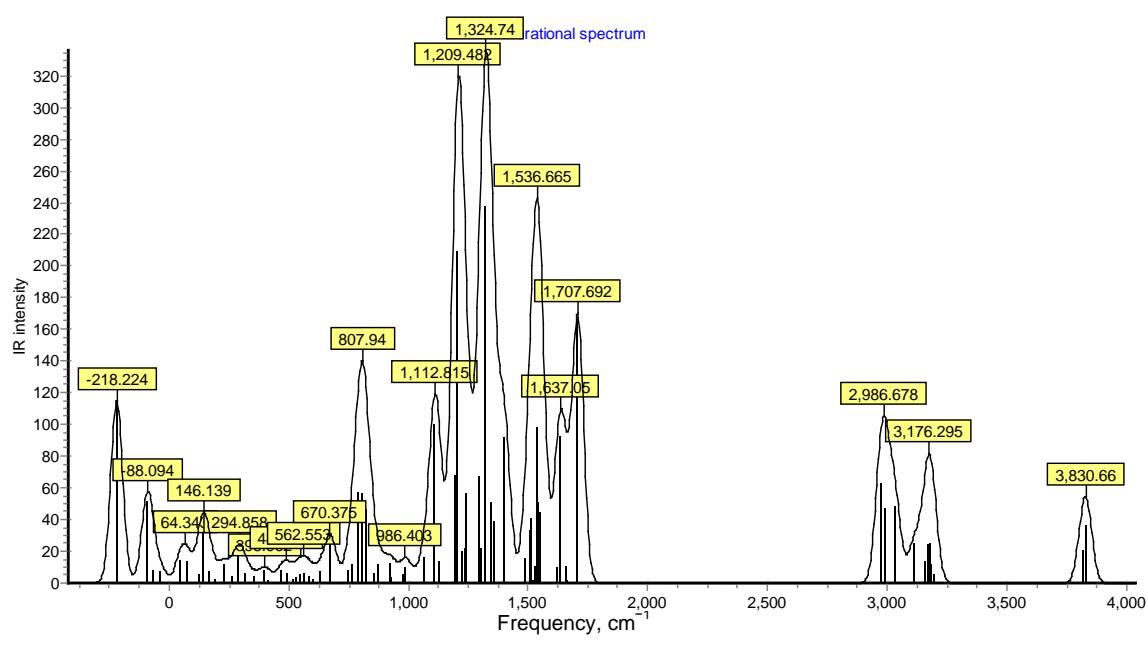

IR SPECTRUM OF VA

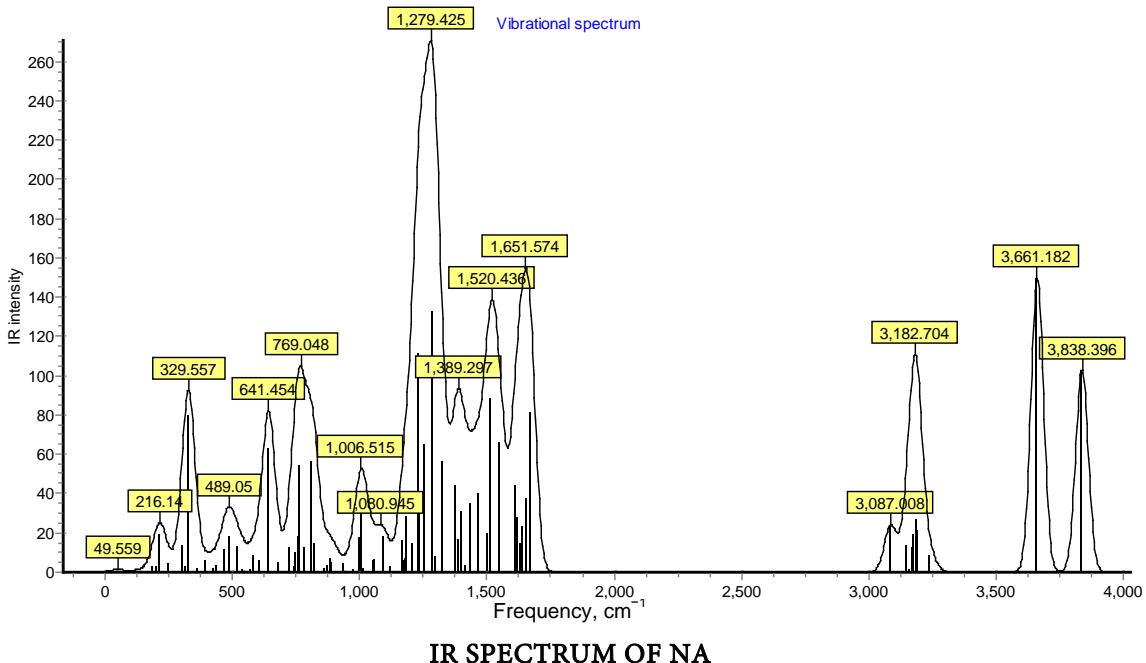

(b)

Figure 1. (a) FT-IR spectrum of salen compounds (Experimental); (b) Vibrational frequency calculation by DFT method.

Table 1. Lemental analysis of Salen compounds.

\begin{tabular}{|c|c|c|c|c|c|c|}
\hline \multirow{2}{*}{$\begin{array}{l}\dot{0} \\
\dot{z}\end{array}$} & \multirow{2}{*}{ 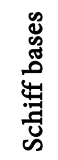 } & \multirow{2}{*}{ Molecular formulae } & \multirow{2}{*}{$\begin{array}{c}\text { Melting Point } \\
\left({ }^{\circ} \mathrm{C}\right)\end{array}$} & \multicolumn{3}{|c|}{ Elemental Analysis-Found (Calc.) } \\
\hline & & & & $\mathrm{C}$ & $\mathrm{H}$ & $\mathrm{N}$ \\
\hline 1. & SA & $\mathrm{C}_{13} \mathrm{H}_{11} \mathrm{O}_{2} \mathrm{~N}$ & $150.1-199.3$ & $73.01(73.23)$ & $5.06(5.20)$ & $6.51(6.57)$ \\
\hline 2. & $\mathrm{AA}$ & $\mathrm{C}_{14} \mathrm{H}_{13} \mathrm{O}_{2} \mathrm{~N}$ & $143.6-164.0$ & $73.83(73.99)$ & $5.59(5.77)$ & $6.20(6.16)$ \\
\hline 3. & VA & $\mathrm{C}_{14} \mathrm{H}_{13} \mathrm{O}_{3} \mathrm{~N}$ & $197.9-221.7$ & $68.78(69.12)$ & $5.20(5.39)$ & $5.65(5.76)$ \\
\hline 4. & NA & $\mathrm{C}_{17} \mathrm{H}_{13} \mathrm{O}_{2} \mathrm{~N}$ & $239.3-245.0$ & 77.46) (77.55) & $5.00(4.98)$ & $5.18(5.32)$ \\
\hline
\end{tabular}

the four compounds has been assigned to phenolic C-O stretching and a band due to $\mathrm{ph}-\mathrm{N}=\mathrm{C}$ - of aminophenol group exhibited in the region $1274-1296 \mathrm{~cm}^{-1}$. Further the spectra of all the Schiff bases showed a weak band in the 3020-3069 $\mathrm{cm}^{-1}$ region due to $-\mathrm{N}=\mathrm{CH}-/ \mathrm{N}=\mathrm{C}\left(\mathrm{CH}_{3}\right)$ - groups of NA, SA, VA and AA [14]. 
Theoretically all the fundamental vibrations were active in IR. The results showed that the DFT (B3LYP) method applied in this work leads to vibrational wavenumbers which are in good agreements with the experimental data. It is noteworthy that the very important role of vibrational frequencies salen compounds, based on experimental data as well as theoretical calculations, the computed vibrational frequencies in Table 2 and Figure 1 for SA, AA, VA and NA at the DFT level of theory are in acceptable agreement with the experimental data.

\subsubsection{UV-Vis Spectra}

The UV-Visible spectra of the salen compounds (Figure 2) exhibit mainly two bands. The first band on the higher energy side, the range $\lambda_{\max }=350-385 \mathrm{~nm}$, due to the excitation of the $\pi$-electrons ( $\pi \rightarrow \pi^{*}$ transitions) of the aromatic azo-
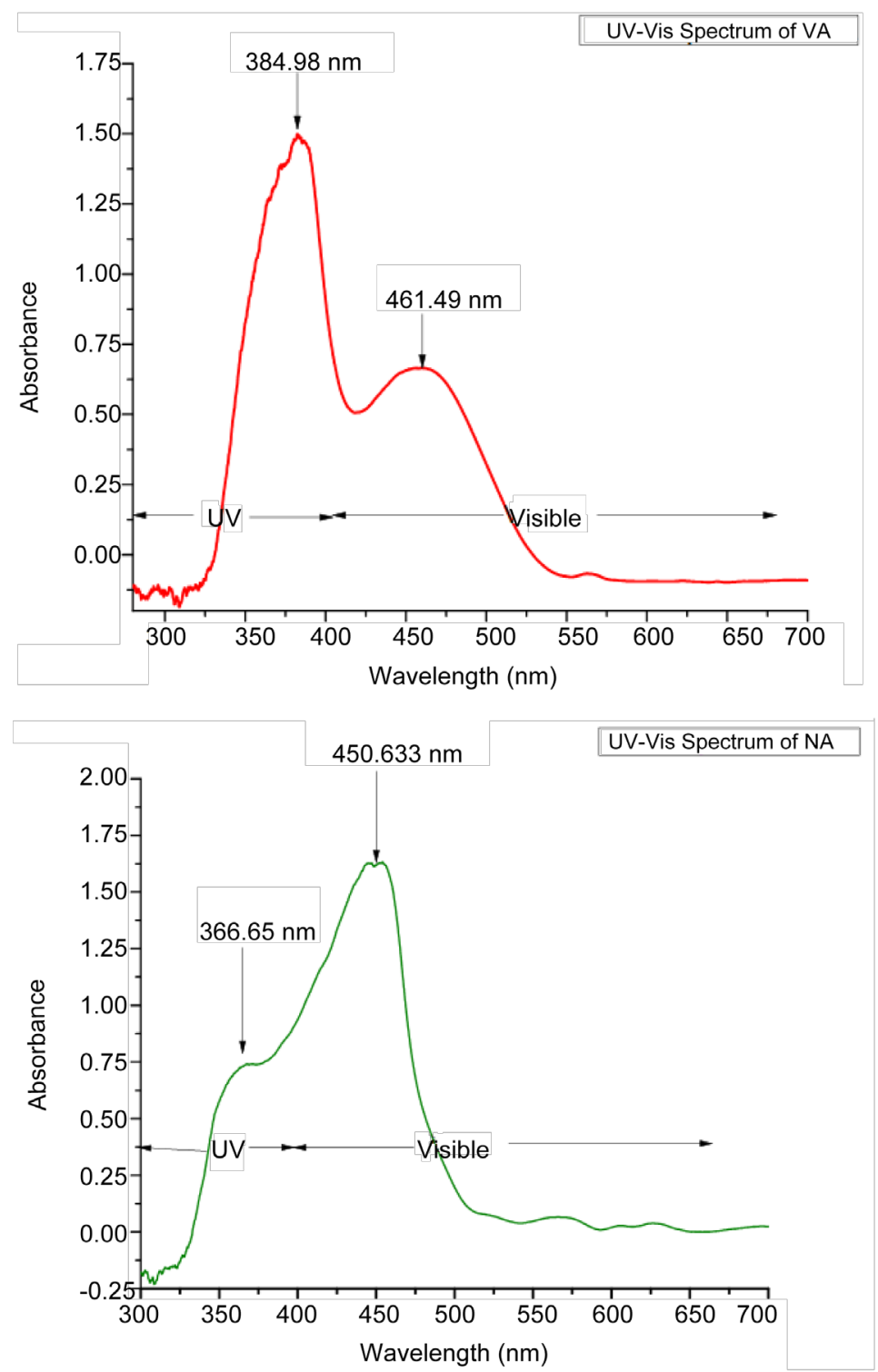

Figure 2. UV-Vis Spectra. 
Table 2. Vibrational frequencies of salen compounds (Experimental \& Theoretical).

\begin{tabular}{|c|c|c|c|c|c|c|c|c|c|c|c|}
\hline \multirow{3}{*}{$\begin{array}{l}\dot{0} \\
\dot{Z} \\
\dot{s}\end{array}$} & \multirow{3}{*}{ 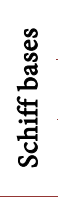 } & \multicolumn{10}{|c|}{ FT-IR $\left(\mathrm{cm}^{-1}\right)$} \\
\hline & & \multicolumn{5}{|c|}{ Experimental } & \multicolumn{5}{|c|}{ DFT (B3LYP/6-311G $(\mathrm{d}, \mathrm{p}))$} \\
\hline & & $v_{>C=N-}$ & $v_{\mathrm{ph}-\mathrm{OH}}$ & $v_{\mathrm{ph}-\mathrm{C}-\mathrm{O}}$ & $v_{\mathrm{ph}-\mathrm{N}=\mathrm{C}-}$ & $\begin{array}{c}v-\mathrm{N}=\mathrm{CH}-/ \\
v-\mathrm{N}=\mathrm{C}(\mathrm{CH} 3)-\end{array}$ & $v_{>\mathrm{C}=\mathrm{N}-}$ & $v_{\mathrm{ph}-\mathrm{OH}}$ & $\begin{array}{c}v_{\text {ph-C-O } /} \\
v_{\text {Ph-OCH3- }}\end{array}$ & $v_{\mathrm{ph}-\mathrm{N}=\mathrm{C}<}$ & $\begin{array}{c}v-\mathrm{N}=\mathrm{CH}-l \\
v-\mathrm{N}=\mathrm{C}(\mathrm{CH} 3)-\end{array}$ \\
\hline 1. & SA & 1671 & 3363 & 1367 & 1274 & 3020 & 1663 & 3832 & 1305 & 1199 & 3178 \\
\hline 2. & AA & 1656 & 3399 & 1362 & 1276 & 3054 & 1685 & 3833 & 1321 & 1253 & 3178 \\
\hline 3. & VA & 1692 & 3375 & 1371 & 1281 & 3029 & 1708 & 3831 & 1325 & 1209 & 2987 \\
\hline 4. & NA & 1642 & 3308 & 1373 & 1296 & 3069 & 1652 & 3838 & 1389 & 1279 & 3183 \\
\hline
\end{tabular}

methine group. The second band, $\lambda_{\max }=450-480 \mathrm{~nm}$ range is assigned to an intramolecular charge transfer (ICT transition) involving the salen compounds. This band observed in salicylaldimine compounds of salen derivatives is facilitated by the presence of intramolecular hydrogen bonding between the $-\mathrm{OH}$ group and the azomethine nitrogen [26].

\subsection{3. ${ }^{13} \mathrm{C}\left\{{ }^{1} \mathrm{H}\right\}$-NMR Spectra}

The formations of Schiff base were conveniently monitored by peak ratios in the ${ }^{1} \mathrm{H}-\mathrm{NMR}$ spectra. ${ }^{1} \mathrm{H}-\mathrm{NMR}$ spectra of all the four compounds (Table 3 and Figure 3) were taken in DMSO.d $\mathrm{d}_{6}$ solvent. The aromatic region was a set of multiplets in the range $6.6-8.4 \mathrm{ppm}$ for all the Schiff bases, while the azomethine proton of SA, VA and NA compounds were observed in the range $9.0-9.5 \mathrm{ppm}$. The phenolic-OH protons of all the four Schiff bases were observed as a singlet in the region 13.8-15.6 ppm. ${ }^{1} \mathrm{H}-\mathrm{NMR}$ spectra exhibited a strong peak at 3.8 and $3.5 \mathrm{ppm}$ for $-\mathrm{OCH}_{3}$ protons of $\mathrm{VA}$ and $-\mathrm{CH}_{3}$ protons of AA salen compounds.

${ }^{13} \mathrm{C}$-NMR spectral data (Table 3 and Figure 3 ) were consistent with ${ }^{1} \mathrm{H}-\mathrm{NMR}$ spectral data. The methyl carbon of aliphatic substituents, azomethine $(>\mathrm{C}=\mathrm{N})$ for all the Schiff bases and for Ph-C- $\mathrm{CH}_{3}$ of AA and $\mathrm{Ph}-\mathrm{OCH}_{3}$ of VA compounds were 45 and $56 \mathrm{ppm}$ respectively. The resonance observed at $108-178 \mathrm{ppm}$ was assigned to the phenyl group carbon of salen compounds.

\subsection{DFT Study}

\subsubsection{Conformational Analysis and Optimized Geometries}

At the minimum energy conformations (Figure 4) obtained from the energy scan, further geometry optimization was performed with the B3LYP/6-311G $(d, p)$ basis set. No geometrical parameter constraint was imposed during the optimization, except those favoring the stabilizing effects due to hydrogen bonding between two adjacent -OH groups. The optimized structures of the most stable conformers of neutral form of salen compounds are shown in Figure 5.

The optimized geometrical parameters are shown in Table 4 (Supplement materials). From the data of bond distances and bond angles, it can be seen that no significant geometrical change has been observed for all the compounds. From the data of dihedral angle in Table 4, it can also be seen that compounds with o-hydroxyl group in A-ring are completely planar, while others have some 

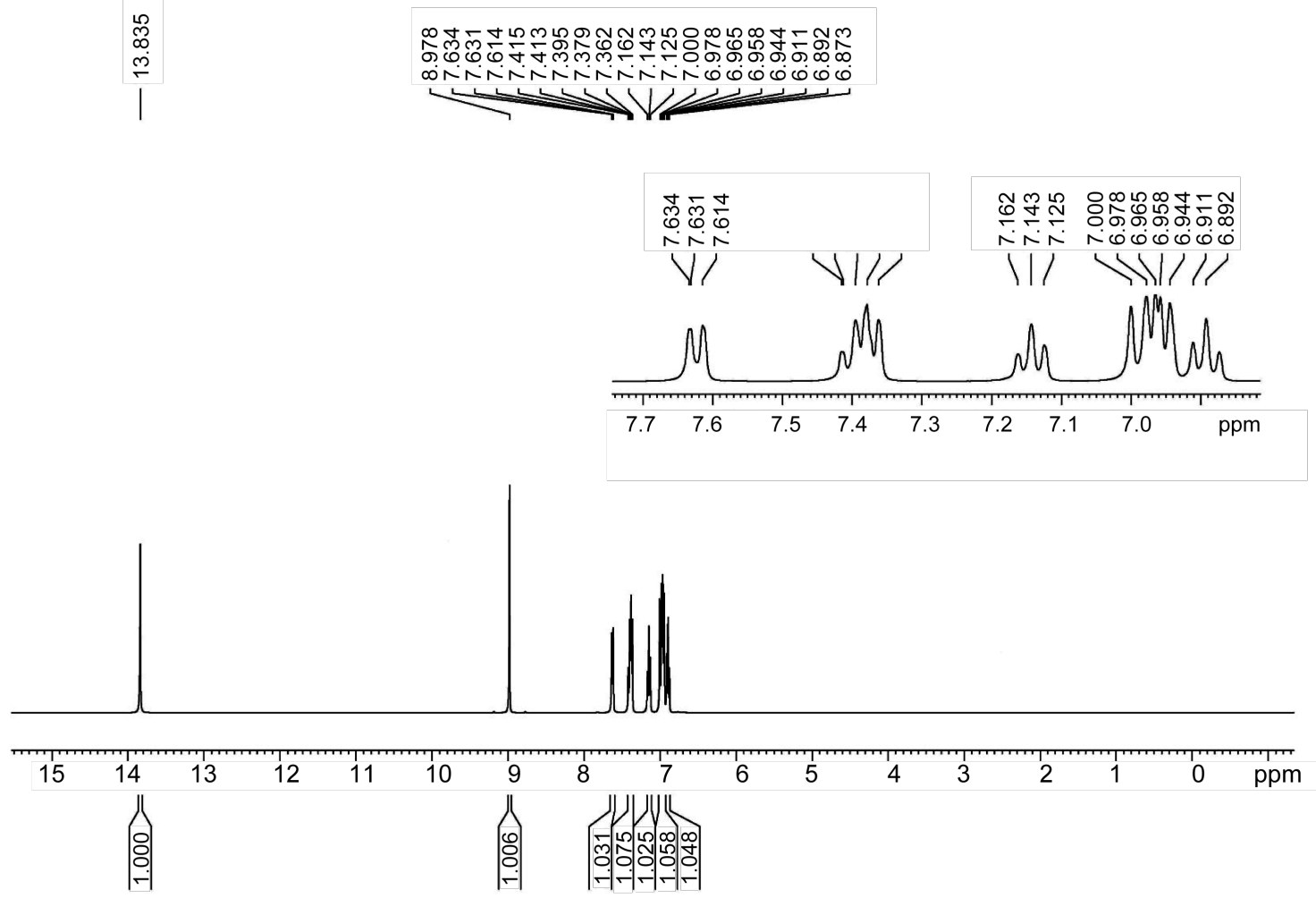

${ }^{1} \mathrm{H}$-NMR Spectrum of SA

${ }^{1} \mathrm{H}-\mathrm{NMR}$ Spectrum of CA
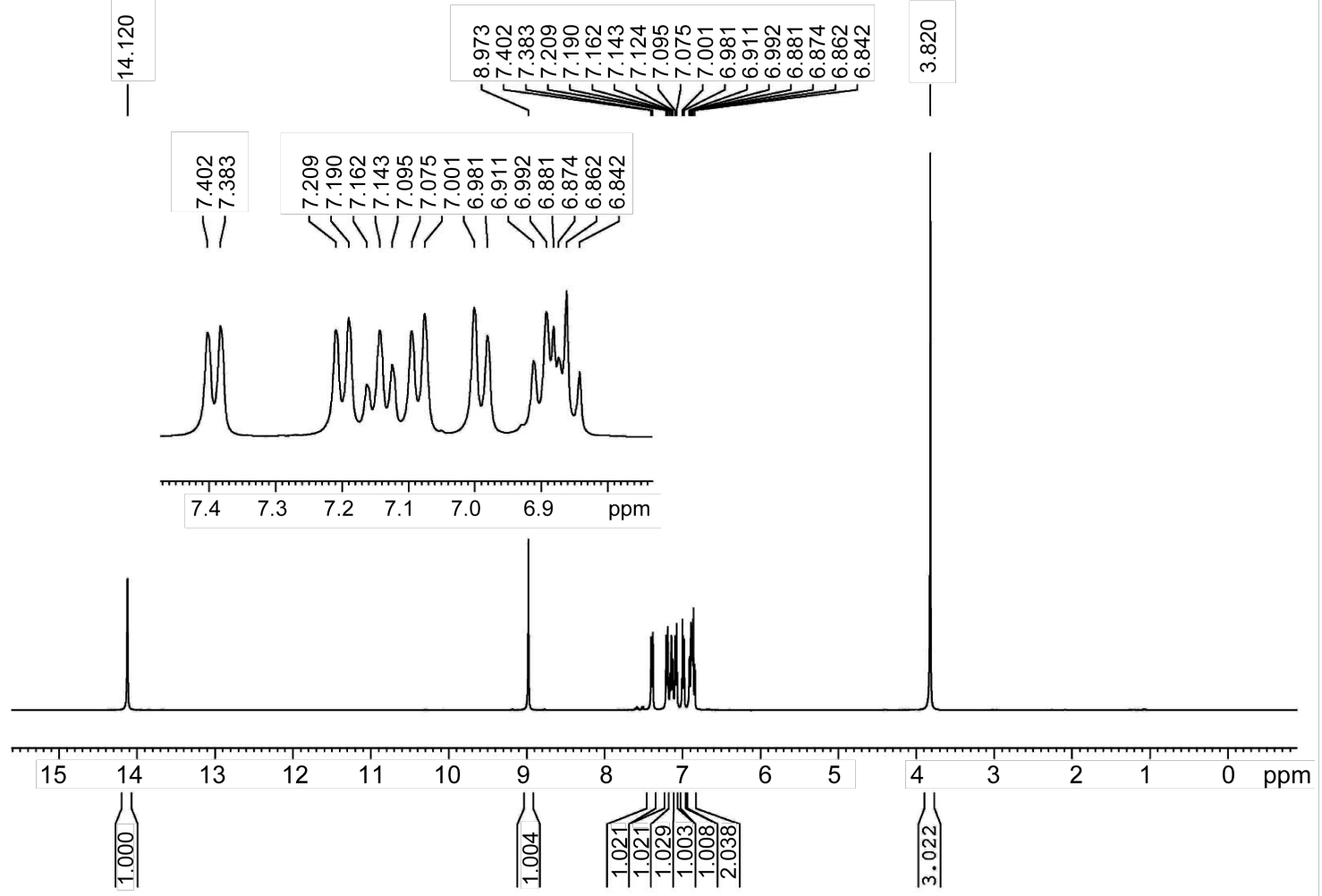

${ }^{1} \mathrm{H}-\mathrm{NMR}$ Spectrum of VA 

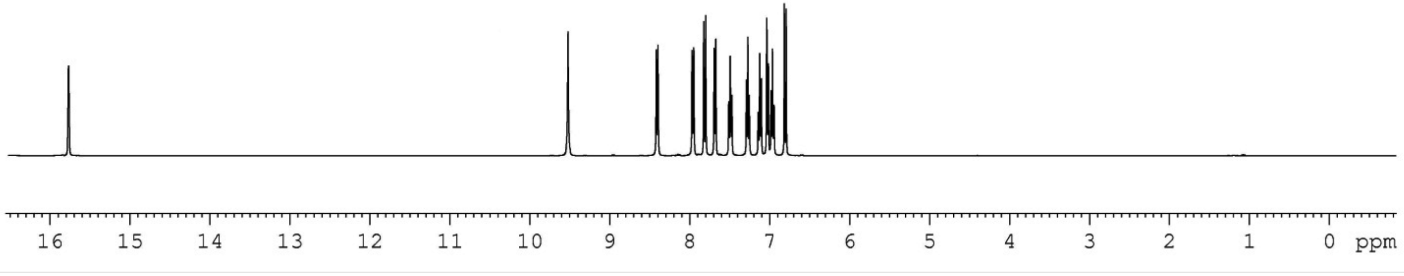

${ }^{1} \mathrm{H}-\mathrm{NMR}$ Spectrum of NA

(a)
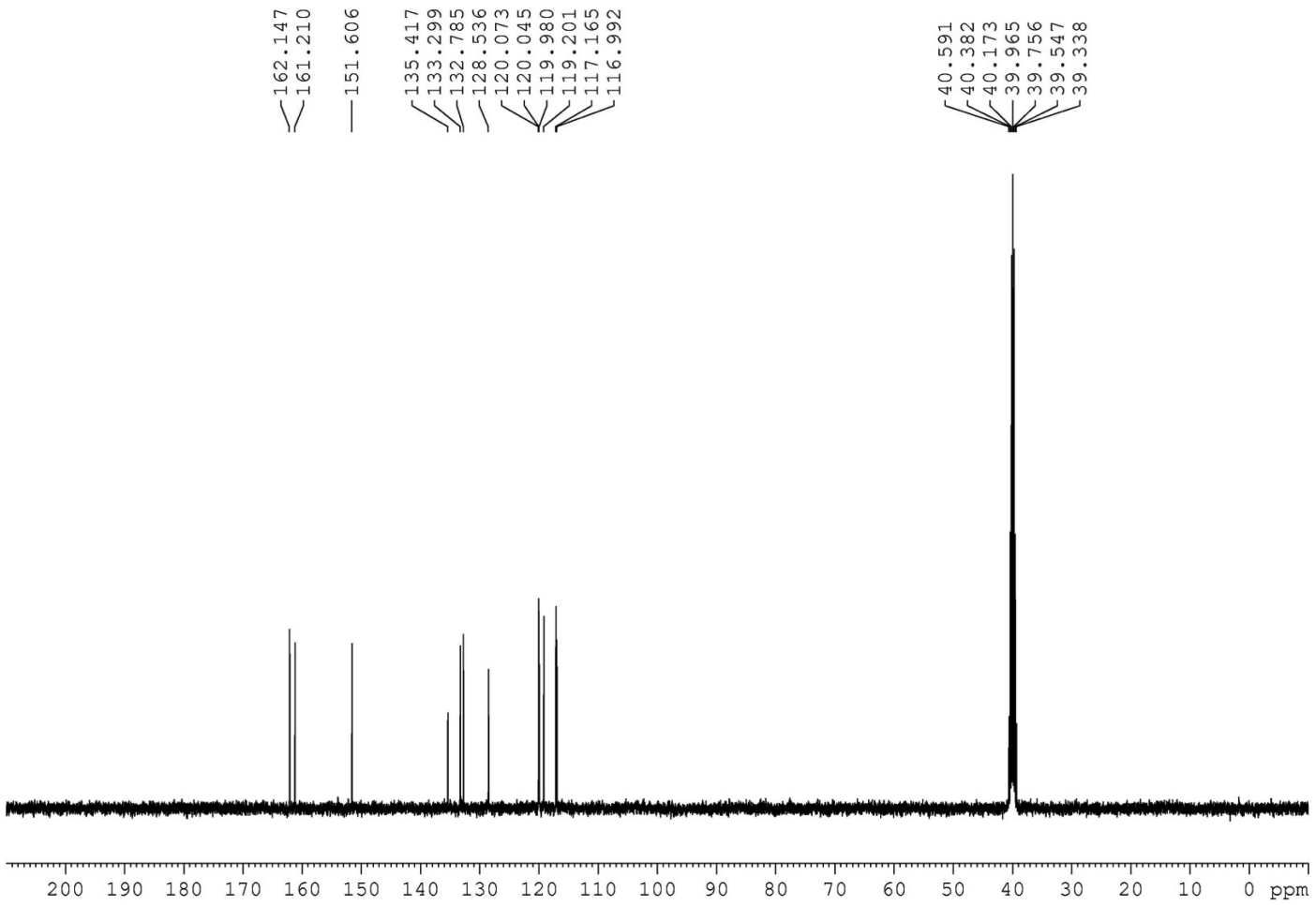

${ }^{13}$ C-NMR Spectrum of SA 
${ }^{13} \mathrm{C}-\mathrm{NMR}$ Spectrum of VA

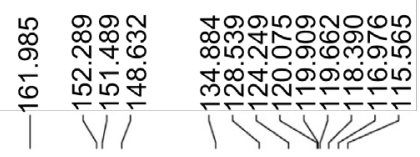

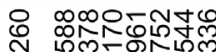

เ

1
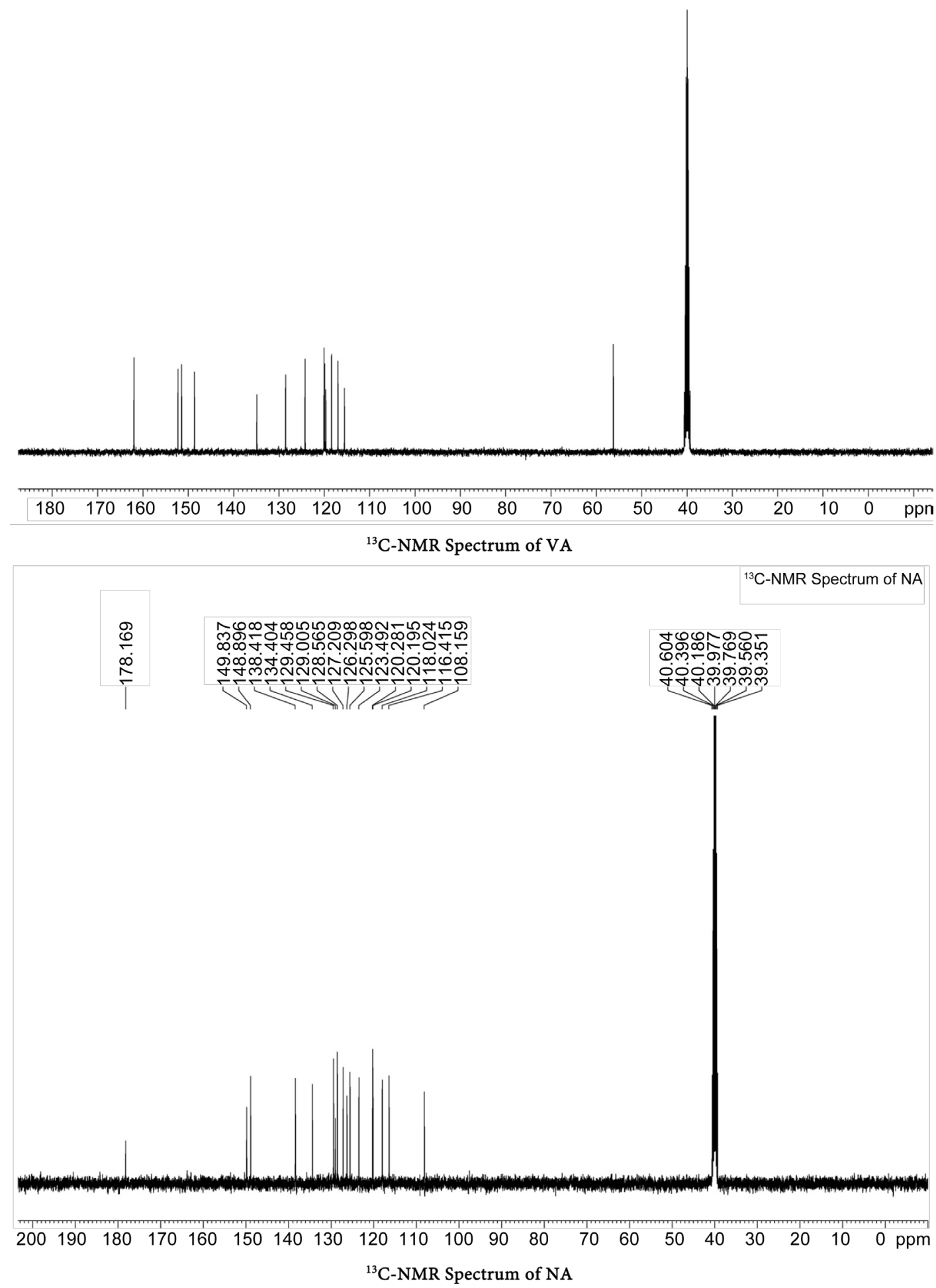

(b)

Figure 3. (a) ${ }^{1} \mathrm{H}$-NMR Spectra of salen compounds, (b) ${ }^{13} \mathrm{C}$-NMR Spectra of salen compounds. 


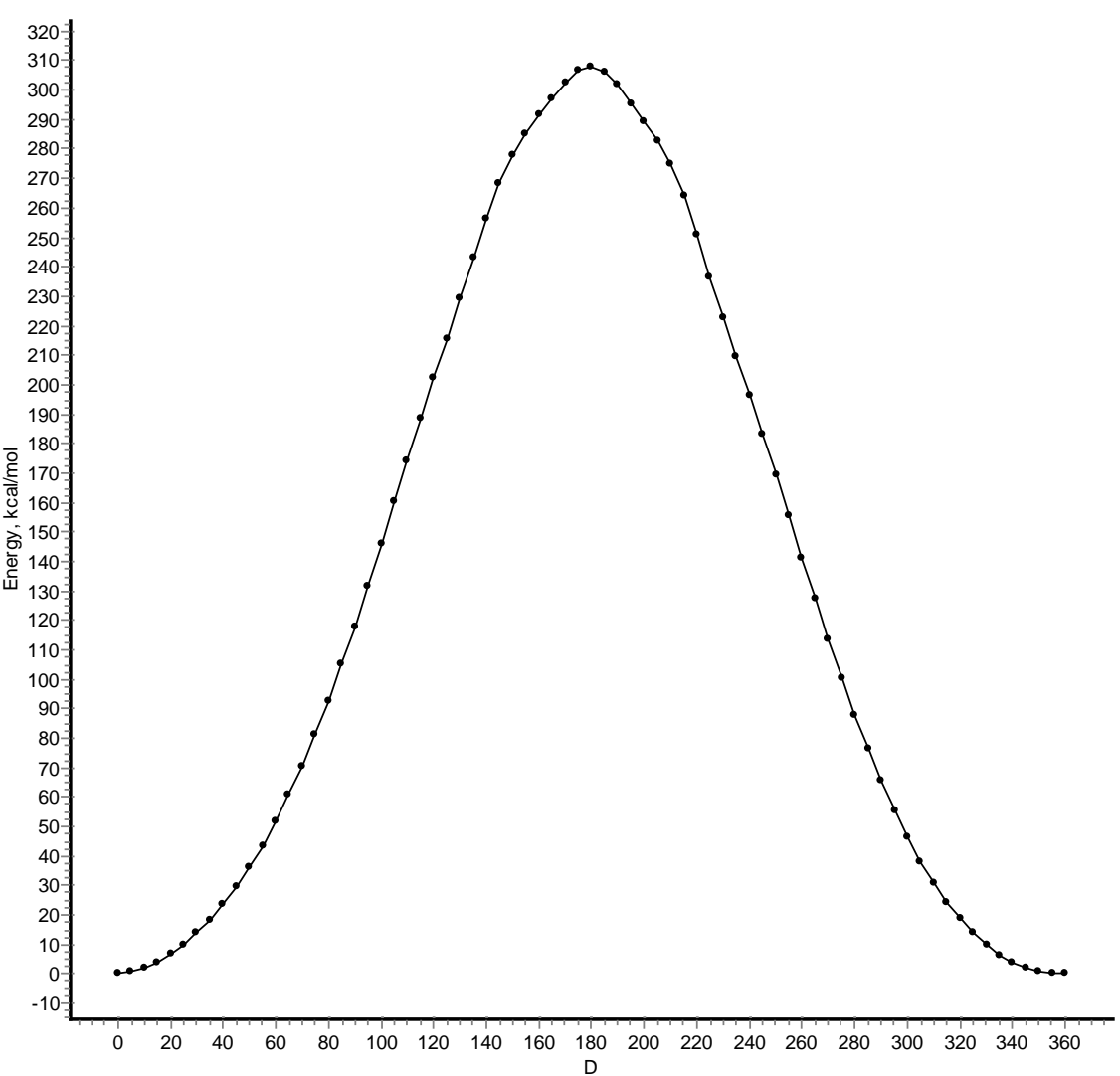

Figure 4. PES scan optimization of salen compound AA.

Table 3. NMR Chemical Shifting of Salen compounds.

\begin{tabular}{|c|c|c|c|}
\hline \multirow{2}{*}{$\begin{array}{l}\dot{a} \\
\dot{z}\end{array}$} & \multirow{2}{*}{ 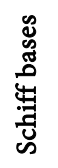 } & \multicolumn{2}{|c|}{ NMR Spectral data (Experimental) } \\
\hline & & ${ }^{1} \mathrm{H}$ & ${ }^{13} \mathrm{C}$ \\
\hline 1. & SA & $\begin{array}{c}6.9-7.6 \mathrm{~m} \text {, aromatic protons, } \\
9.0 \mathrm{~s}-\mathrm{CH}=\mathrm{N}-, 13.8 \mathrm{~s} \text { ph-OH }\end{array}$ & $\begin{array}{c}120.0-160.6 \mathrm{ppm} \text { phenyl Carbons, } \\
18.9 \mathrm{ppm}-\mathrm{CH}=\mathrm{N} \text { carbon }\end{array}$ \\
\hline 2. & AA & $\begin{array}{l}6.6-7.3 \mathrm{~m} \text {, aromatic protons, } \\
9.0 \mathrm{~s} \mathrm{ph}-\mathrm{OH}, 3.5 \mathrm{~s}-\mathrm{CH}_{3} \text { protons }\end{array}$ & $\begin{array}{c}160 \text { - } 180.6 \text { ppm phenyl Carbons, } \\
19.0 \text { ppm }-\mathrm{C}-\mathrm{CH}_{3}\end{array}$ \\
\hline 3. & VA & $\begin{array}{c}6.8-7.4 \mathrm{~m} \text {, aromatic protons, } 9.0 \mathrm{~s}-\mathrm{CH}=\mathrm{N}-\text {, } \\
14.1 \mathrm{~s} \text { ph- } \mathrm{OH}, 3.8 \mathrm{~s}-\mathrm{OCH}_{3} \text { protons }\end{array}$ & $\begin{array}{c}146-178.0 \mathrm{ppm} \text { phenyl Carbons, } \\
19.5 \mathrm{ppm}-\mathrm{CH}=\mathrm{N} \text { - and }-\mathrm{OCH}_{3}\end{array}$ \\
\hline 4. & NA & $\begin{array}{c}6.8-8.4 \mathrm{~m} \text {, aromatic protons, } \\
9.5 \mathrm{~s}-\mathrm{CH}=\mathrm{N}-, 15.8 \mathrm{~s} \mathrm{ph}-\mathrm{OH}\end{array}$ & $\begin{array}{l}160-180.6 \mathrm{ppm} \text { phenyl and naphtyl } \\
\text { Carbons, } 19.6 \mathrm{ppm}-\mathrm{CH}=\mathrm{N} \text {-carbon }\end{array}$ \\
\hline
\end{tabular}

degree of deviation from the planarity due to the torsion between A-ring and the plane of aminophenol system B-ring (Scheme 2).

In Table 5 the dipole moment of four compounds at B3LYP/6-311G(d,p) level of computation have been reported. The Dipole moment values lie in the range of $1.7675 \mathrm{D}$ to $4.8823 \mathrm{D}$ for salen compounds. These values are rather high, reflecting the numerous polarized hydroxyl or carbonyl functions distributed over the structures. The three structures of salen compounds differ only by the presence of methyl at C7, methoxy at C3 and naphthyl groups in the A-ring and there is a little difference in the dipole moment value. The molecular dipole moment is used to measure bond polarities and charge densities in a molecule. 


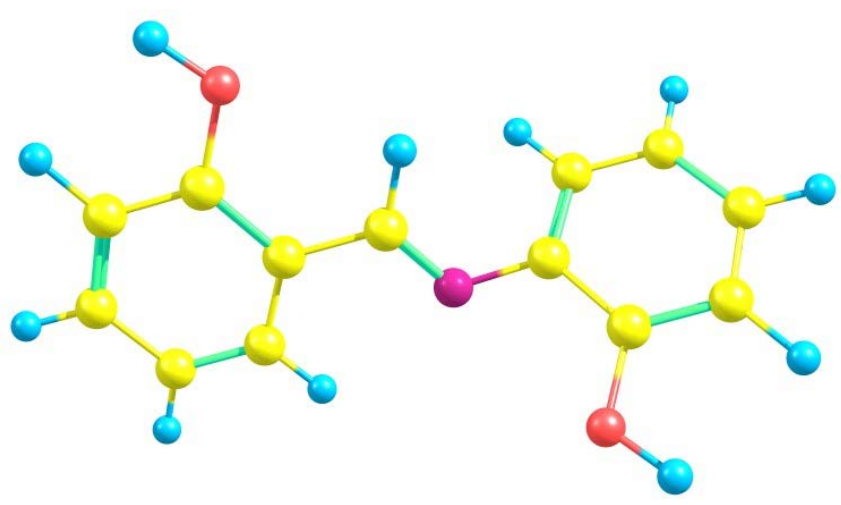

SA

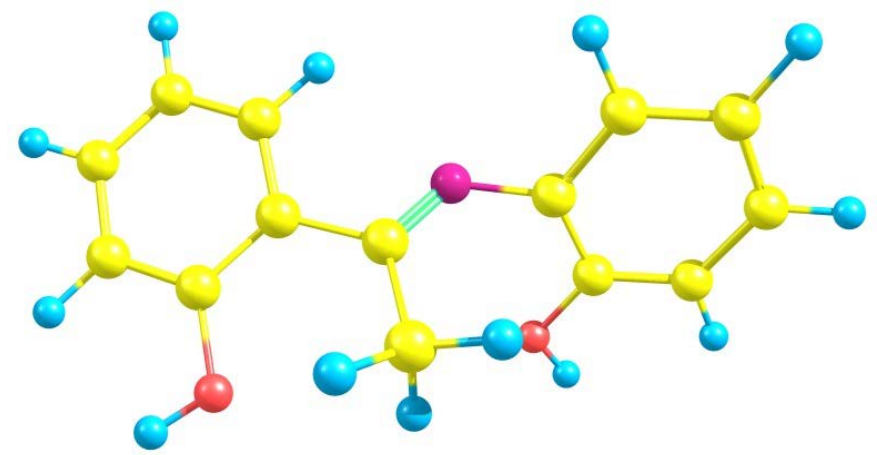

AA

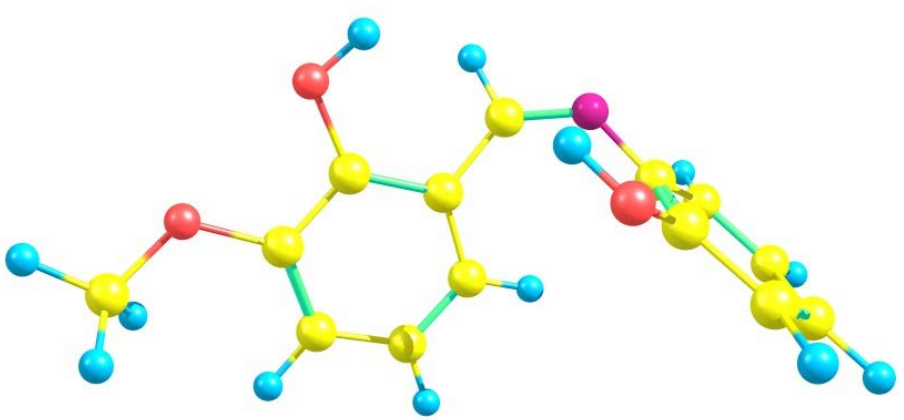

VA

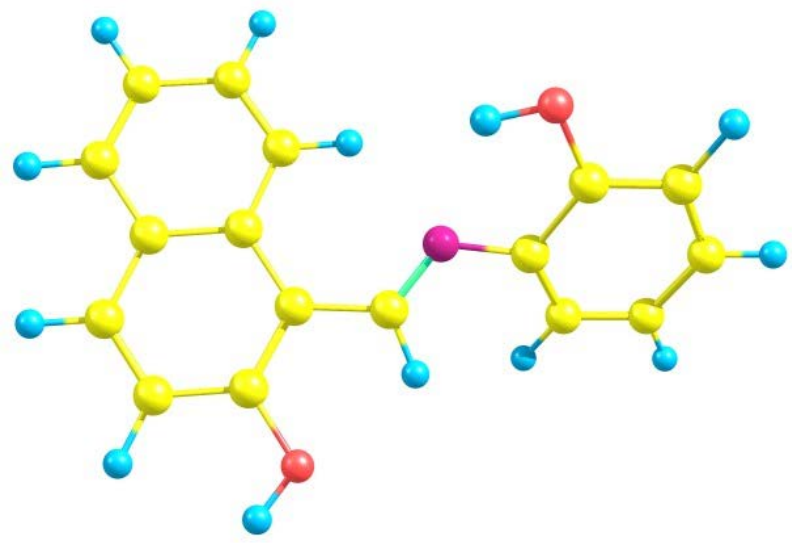

NA

Figure 5. Optimized structure of Salen compounds with the B3LYP/6-311G(d,p) basis set. 
Table 4. Geometry parameters of Schiff bases and its radicals at B3LYP/6-311G(d,p) (Bond distances and Bond Angle in $\AA$ ).

\begin{tabular}{|c|c|c|}
\hline \multirow{2}{*}{ Bond parameters } & \multicolumn{2}{|c|}{ SA } \\
\hline & Neutral & Diradical \\
\hline $\mathrm{C} 1-\mathrm{C} 2$ & $1 . .408$ & 1.482 \\
\hline $\mathrm{C} 2-\mathrm{C} 3$ & 1.396 & 1.459 \\
\hline $\mathrm{C} 3-\mathrm{C} 4$ & 1.390 & 1.358 \\
\hline C4-C5 & 1.396 & 1.439 \\
\hline $\mathrm{C} 5-\mathrm{C} 6$ & 1.387 & 1.359 \\
\hline $\mathrm{C} 6-\mathrm{C} 1$ & 1.403 & 1.436 \\
\hline $\mathrm{C} 2-\mathrm{O} 1$ & 1.367 & 1.234 \\
\hline $\mathrm{O} 1-\mathrm{H} 5$ & 0.963 & - \\
\hline $\mathrm{C} 1-\mathrm{C} 7$ & 1.467 & 1.376 \\
\hline C7-H6 & 1.094 & 1.092 \\
\hline $\mathrm{C} 7-\mathrm{N} 1$ & 1.278 & 1.340 \\
\hline N1-C8 & 1.400 & 1.280 \\
\hline C8-C9 & 1.413 & 1.528 \\
\hline C9-C10 & 1.393 & 1.472 \\
\hline $\mathrm{C} 10-\mathrm{C} 11$ & 1.394 & 1.348 \\
\hline $\mathrm{C} 11-\mathrm{C} 12$ & 1.391 & 1,454 \\
\hline $\mathrm{C} 12-\mathrm{C} 13$ & 1.393 & 1.349 \\
\hline $\mathrm{C} 13-\mathrm{C} 8$ & 1.401 & 1.456 \\
\hline $\mathrm{C} 9-\mathrm{O} 2$ & 1.363 & 1.214 \\
\hline $\mathrm{O} 2-\mathrm{H} 11$ & 0.963 & - \\
\hline $\mathrm{C} 6-\mathrm{C} 1-\mathrm{C} 2$ & 118.35 & 120.47 \\
\hline $\mathrm{C} 1-\mathrm{C} 2-\mathrm{C} 3$ & 120.37 & 115.51 \\
\hline $\mathrm{C} 2-\mathrm{C} 3-\mathrm{C} 4$ & 120.11 & 121.15 \\
\hline $\mathrm{C} 3-\mathrm{C} 4-\mathrm{C} 5$ & 120.26 & 122.19 \\
\hline C4-C5-C6 & 119.51 & 120.00 \\
\hline C5-C6-C1 & 121.41 & 120.51 \\
\hline $\mathrm{C} 1-\mathrm{C} 2-\mathrm{O} 1$ & 117.89 & 121.23 \\
\hline $\mathrm{C} 3-\mathrm{C} 2-\mathrm{O} 1$ & 121.75 & 123.26 \\
\hline $\mathrm{C} 2-\mathrm{O} 1-\mathrm{H} 5$ & 109.23 & - \\
\hline $\mathrm{C} 2-\mathrm{C} 1-\mathrm{C} 7$ & 120.45 & 118.16 \\
\hline C6-C1-C7 & 121.20 & 121.35 \\
\hline C1-C7-H6 & 116.26 & 118.70 \\
\hline C1-C7-N1 & 121.68 & 125.96 \\
\hline $\mathrm{H} 6-\mathrm{C} 7-\mathrm{N} 1$ & 122.06 & 114.94 \\
\hline C7-N1-C8 & 120.11 & 132.23 \\
\hline
\end{tabular}




\section{Continued}

\begin{tabular}{ccc}
\hline N1-C8-C9 & 117.52 & 117.14 \\
N1-C8-C13 & 123.42 & 124.00 \\
C8-C9-C10 & 120.00 & 115.86 \\
C9-C10-C11 & 120.72 & 121.35 \\
C10-C11-C12 & 119.88 & 121.87 \\
C11-C12-C13 & 119.59 & 121.60 \\
C12-C13-C8 & 121.54 & 120.45 \\
C13-C8-C9 & 117.52 & 118.83 \\
C8-C9-O2 & 122.46 & 121.49 \\
C9-O2-H11 & 108.61 & - \\
C10-C9-O2 & 122.46 & 122.46 \\
\hline
\end{tabular}

\begin{tabular}{|c|c|c|}
\hline \multirow{2}{*}{ Bond parameters } & \multicolumn{2}{|c|}{ AA } \\
\hline & Neutral & Diradical \\
\hline $\mathrm{C} 1-\mathrm{C} 2$ & 1.408 & 1.493 \\
\hline $\mathrm{C} 2-\mathrm{C} 3$ & 1.398 & 1.462 \\
\hline $\mathrm{C} 3-\mathrm{C} 4$ & 1.388 & 1.355 \\
\hline $\mathrm{C} 4-\mathrm{C} 5$ & 1.394 & 1.438 \\
\hline C5-C6 & 1.387 & 1.358 \\
\hline $\mathrm{C} 6-\mathrm{C} 1$ & 1.403 & 1.441 \\
\hline $\mathrm{C} 2-\mathrm{O} 1$ & 1.368 & 1.235 \\
\hline O1-H11 & 0.963 & - \\
\hline $\mathrm{C} 1-\mathrm{C} 7$ & 1.497 & 1.394 \\
\hline C7-N1 & 1.280 & 1.363 \\
\hline N1-C8 & 1.402 & 1.286 \\
\hline C8-C9 & 1.411 & 1.533 \\
\hline $\mathrm{C} 9-\mathrm{C} 10$ & 1.394 & 1.472 \\
\hline $\mathrm{C} 10-\mathrm{C} 11$ & 1.393 & 1.348 \\
\hline $\mathrm{C} 11-\mathrm{C} 12$ & 1.392 & 1.454 \\
\hline $\mathrm{C} 12-\mathrm{C} 13$ & 1.391 & 1.350 \\
\hline $\mathrm{C} 13-\mathrm{C} 8$ & 1.400 & 1.457 \\
\hline $\mathrm{C} 9-\mathrm{O} 2$ & 1.370 & 1.215 \\
\hline $\mathrm{O} 2-\mathrm{H} 10$ & 0.962 & - \\
\hline $\mathrm{C} 7-\mathrm{C} 14$ & 1.511 & 1.507 \\
\hline $\mathrm{C} 6-\mathrm{C} 1-\mathrm{C} 2$ & 117.41 & 118.94 \\
\hline $\mathrm{C} 1-\mathrm{C} 2-\mathrm{C} 3$ & 120.44 & 115.85 \\
\hline $\mathrm{C} 2-\mathrm{C} 3-\mathrm{C} 4$ & 120.69 & 121.97 \\
\hline $\mathrm{C} 3-\mathrm{C} 4-\mathrm{C} 5$ & 119.75 & 121.34 \\
\hline
\end{tabular}




\section{Continued}

\begin{tabular}{lll}
\hline C4-C5-C6 & 119.39 & 120.33 \\
C5-C6-C1 & 122.28 & 121.53 \\
C1-C2-O1 & 119.18 & 123.16 \\
C3-C2-O1 & 120.33 & 120.99 \\
C2-O1-H5 & 108.77 & - \\
C2-C1-C7 & 123.89 & 120.90 \\
C6-C1-C7 & 118.69 & 120.12 \\
C1-C7-C14 & 119.60 & 124.05 \\
C1-C7-N1 & 116.03 & 117.52 \\
C14-C7-N1 & 124.28 & 118.29 \\
C7-N1-C8 & 123.73 & 128.59 \\
N1-C8-C9 & 122.39 & 115.98 \\
N1-C8-C13 & 119.33 & 125.75 \\
C8-C9-C10 & 120.27 & 116.30 \\
C9-C10-C11 & 120.73 & 121.26 \\
C10-C11-C12 & 119.61 & 121.69 \\
C11-C12-C13 & 119.74 & 121.88 \\
C12-C13-C8 & 121.65 & 120.59 \\
C8-C9-O2 & 117.53 & 121.40 \\
C10-C9-O2 & 122.19 & - \\
C9-O2-H10 & 108.76 & \\
\hline & & \\
\hline
\end{tabular}

\begin{tabular}{lcc}
\hline & & \\
\hline & & VA \\
\cline { 2 - 3 } Bond parameters & Neutral & Diradical \\
\hline C1-C2 & 1.406 & 1.478 \\
C2-C3 & 1.414 & 1.476 \\
C3-C4 & 1.389 & 1.367 \\
C4-C5 & 1.401 & 1.428 \\
C5-C6 & 1.379 & 1.363 \\
C6-C1 & 1.411 & 1.427 \\
C2-O1 & 1.362 & 1.236 \\
O1-H4 & 0.962 & - \\
C1-C7 & 1.477 & 1.384 \\
C7-H5 & 1.097 & 1.090 \\
C7-N1 & 1.280 & 1.339 \\
N1-C8 & 1.411 & 1.293 \\
C8-C9 & 1.411 & 1.516 \\
C9-C10 & 1.394 & 1.461 \\
\hline & & \\
\hline & & \\
\hline
\end{tabular}




\section{Continued}

\begin{tabular}{|c|c|c|}
\hline $\mathrm{C} 10-\mathrm{C} 11$ & 1.390 & 1.353 \\
\hline $\mathrm{C} 11-\mathrm{C} 12$ & 1.395 & 1.449 \\
\hline $\mathrm{C} 12-\mathrm{C} 13$ & 1.389 & 1.353 \\
\hline $\mathrm{C} 13-\mathrm{C} 8$ & 1.401 & 1.445 \\
\hline $\mathrm{C} 9-\mathrm{O} 2$ & 1.364 & 1.225 \\
\hline $\mathrm{O} 2-\mathrm{H} 10$ & 0.963 & - \\
\hline $\mathrm{C} 3-\mathrm{O} 3$ & 1.358 & 1.357 \\
\hline $\mathrm{O} 3-\mathrm{C} 14$ & 1.421 & 1.436 \\
\hline $\mathrm{C} 6-\mathrm{C} 1-\mathrm{C} 2$ & 119.07 & 121.04 \\
\hline $\mathrm{C} 1-\mathrm{C} 2-\mathrm{C} 3$ & 120.33 & 115.54 \\
\hline $\mathrm{C} 2-\mathrm{C} 3-\mathrm{C} 4$ & 119.27 & 119.62 \\
\hline $\mathrm{C} 3-\mathrm{C} 4-\mathrm{C} 5$ & 120.47 & 122.49 \\
\hline C4-C5-C6 & 120.51 & 120.81 \\
\hline $\mathrm{C} 5-\mathrm{C} 6-\mathrm{C} 1$ & 120.33 & 119.67 \\
\hline $\mathrm{C} 1-\mathrm{C} 2-\mathrm{O} 1$ & 123.97 & 121.19 \\
\hline $\mathrm{C} 3-\mathrm{C} 2-\mathrm{O} 1$ & 115.70 & 123.26 \\
\hline $\mathrm{C} 2-\mathrm{O} 1-\mathrm{H} 4$ & 110.09 & - \\
\hline $\mathrm{C} 2-\mathrm{C} 1-\mathrm{C} 7$ & 118.44 & 117.32 \\
\hline $\mathrm{C} 6-\mathrm{C} 1-\mathrm{C} 7$ & 122.24 & 121.63 \\
\hline C1-C7-H5 & 114.35 & 118.53 \\
\hline C1-C7-N1 & 132.26 & 124.11 \\
\hline $\mathrm{H} 5-\mathrm{C} 7-\mathrm{N} 1$ & 113.29 & 117.09 \\
\hline C7-N1-C8 & 125.67 & 127.71 \\
\hline N1-C8-C9 & 122.89 & 120.95 \\
\hline N1-C8-C13 & 118.12 & 119.77 \\
\hline C8-C9-C10 & 119.81 & 116.19 \\
\hline C9-C10-C11 & 120.69 & 120.84 \\
\hline $\mathrm{C} 10-\mathrm{C} 11-\mathrm{C} 12$ & 120.01 & 122.25 \\
\hline $\mathrm{C} 11-\mathrm{C} 12-\mathrm{C} 13$ & 119.54 & 121.05 \\
\hline $\mathrm{C} 12-\mathrm{C} 13-\mathrm{C} 8$ & 121.29 & 120.37 \\
\hline $\mathrm{C} 8-\mathrm{C} 9-\mathrm{O} 2$ & 122.97 & 120.36 \\
\hline C10-C9-O2 & 117.21 & 123.44 \\
\hline $\mathrm{C} 9-\mathrm{O} 2-\mathrm{H} 10$ & 109.36 & - \\
\hline $\mathrm{C} 2-\mathrm{C} 3-\mathrm{O} 3$ & 115.24 & 121.63 \\
\hline $\mathrm{C} 4-\mathrm{C} 3-\mathrm{O} 3$ & 125.49 & 118.13 \\
\hline $\mathrm{C} 3-\mathrm{O} 3-\mathrm{C} 14$ & 118.15 & 119.07 \\
\hline
\end{tabular}




\begin{tabular}{|c|c|c|}
\hline \multirow{2}{*}{ Bond parameters } & \multicolumn{2}{|c|}{ VA } \\
\hline & Neutral & Diradical \\
\hline $\mathrm{C} 1-\mathrm{C} 2$ & 1.406 & 1.478 \\
\hline $\mathrm{C} 2-\mathrm{C} 3$ & 1.414 & 1.476 \\
\hline $\mathrm{C} 3-\mathrm{C} 4$ & 1.389 & 1.367 \\
\hline $\mathrm{C} 4-\mathrm{C} 5$ & 1.401 & 1.428 \\
\hline C5-C6 & 1.379 & 1.363 \\
\hline $\mathrm{C} 6-\mathrm{C} 1$ & 1.411 & 1.427 \\
\hline $\mathrm{C} 2-\mathrm{O} 1$ & 1.362 & 1.236 \\
\hline $\mathrm{O} 1-\mathrm{H} 4$ & 0.962 & - \\
\hline $\mathrm{C} 1-\mathrm{C} 7$ & 1.477 & 1.384 \\
\hline C7-H5 & 1.097 & 1.090 \\
\hline $\mathrm{C} 7-\mathrm{N} 1$ & 1.280 & 1.339 \\
\hline N1-C8 & 1.411 & 1.293 \\
\hline $\mathrm{C} 8-\mathrm{C} 9$ & 1.411 & 1.516 \\
\hline C9-C10 & 1.394 & 1.461 \\
\hline $\mathrm{C} 10-\mathrm{C} 11$ & 1.390 & 1.353 \\
\hline $\mathrm{C} 11-\mathrm{C} 12$ & 1.395 & 1.449 \\
\hline $\mathrm{C} 12-\mathrm{C} 13$ & 1.389 & 1.353 \\
\hline $\mathrm{C} 13-\mathrm{C} 8$ & 1.401 & 1.445 \\
\hline $\mathrm{C} 9-\mathrm{O} 2$ & 1.364 & 1.225 \\
\hline $\mathrm{O} 2-\mathrm{H} 10$ & 0.963 & - \\
\hline $\mathrm{C} 3-\mathrm{O} 3$ & 1.358 & 1.357 \\
\hline O3-C14 & 1.421 & 1.436 \\
\hline $\mathrm{C} 6-\mathrm{C} 1-\mathrm{C} 2$ & 119.07 & 121.04 \\
\hline $\mathrm{C} 1-\mathrm{C} 2-\mathrm{C} 3$ & 120.33 & 115.54 \\
\hline $\mathrm{C} 2-\mathrm{C} 3-\mathrm{C} 4$ & 119.27 & 119.62 \\
\hline $\mathrm{C} 3-\mathrm{C} 4-\mathrm{C} 5$ & 120.47 & 122.49 \\
\hline $\mathrm{C} 4-\mathrm{C} 5-\mathrm{C} 6$ & 120.51 & 120.81 \\
\hline $\mathrm{C} 5-\mathrm{C} 6-\mathrm{C} 1$ & 120.33 & 119.67 \\
\hline $\mathrm{C} 1-\mathrm{C} 2-\mathrm{O} 1$ & 123.97 & 121.19 \\
\hline $\mathrm{C} 3-\mathrm{C} 2-\mathrm{O} 1$ & 115.70 & 123.26 \\
\hline $\mathrm{C} 2-\mathrm{O} 1-\mathrm{H} 4$ & 110.09 & - \\
\hline $\mathrm{C} 2-\mathrm{C} 1-\mathrm{C} 7$ & 118.44 & 117.32 \\
\hline $\mathrm{C} 6-\mathrm{C} 1-\mathrm{C} 7$ & 122.24 & 121.63 \\
\hline C1-C7-H5 & 114.35 & 118.53 \\
\hline C1-C7-N1 & 132.26 & 124.11 \\
\hline $\mathrm{H} 5-\mathrm{C} 7-\mathrm{N} 1$ & 113.29 & 117.09 \\
\hline C7-N1-C8 & 125.67 & 127.71 \\
\hline
\end{tabular}




\section{Continued}

\begin{tabular}{lcc}
\hline N1-C8-C9 & 122.89 & 120.95 \\
N1-C8-C13 & 118.12 & 119.77 \\
C8-C9-C10 & 119.81 & 116.19 \\
C9-C10-C11 & 120.69 & 120.84 \\
C10-C11-C12 & 120.01 & 122.25 \\
C11-C12-C13 & 119.54 & 121.05 \\
C12-C13-C8 & 121.29 & 120.37 \\
C8-C9-O2 & 122.97 & 120.36 \\
C10-C9-O2 & 117.21 & 123.44 \\
C9-O2-H10 & 109.36 & - \\
C2-C3-O3 & 115.24 & 121.63 \\
C4-C3-O3 & 125.49 & 118.13 \\
C3-O3-C14 & 118.15 & 119.07 \\
\hline
\end{tabular}

\begin{tabular}{|c|c|c|}
\hline \multirow{2}{*}{ Bond parameters } & \multicolumn{2}{|c|}{ NA } \\
\hline & Neutral & Diradical \\
\hline $\mathrm{C} 1-\mathrm{C} 2$ & 1.399 & 1.386 \\
\hline $\mathrm{C} 2-\mathrm{C} 3$ & 1.411 & 1.403 \\
\hline $\mathrm{C} 3-\mathrm{C} 4$ & 1.368 & 1.370 \\
\hline $\mathrm{C} 4-\mathrm{C} 5$ & 1.417 & 1.420 \\
\hline C5-C6 & 1.433 & 1.432 \\
\hline C6-C1 & 1.444 & 1.431 \\
\hline $\mathrm{C} 2-\mathrm{O} 1$ & 1.365 & 1.356 \\
\hline O1-H7 & 0.962 & - \\
\hline C6-C14 & 1.420 & 1.410 \\
\hline $\mathrm{C} 14-\mathrm{C} 15$ & 1.377 & 1.376 \\
\hline $\mathrm{C} 15-\mathrm{C} 16$ & 1.410 & 1.411 \\
\hline $\mathrm{C} 16-\mathrm{C} 17$ & 1.373 & 1.374 \\
\hline $\mathrm{C} 17-\mathrm{C} 5$ & 1.418 & 1.417 \\
\hline $\mathrm{C} 1-\mathrm{C} 7$ & 1.461 & 1.456 \\
\hline $\mathrm{C} 7-\mathrm{H} 8$ & 1.092 & 1.088 \\
\hline C7-N1 & 1.285 & 1.280 \\
\hline N1-C8 & 1.403 & 1.446 \\
\hline $\mathrm{O} 1-\mathrm{C} 8$ & - & 1.455 \\
\hline $\mathrm{C} 8-\mathrm{C} 9$ & 1.414 & 1.570 \\
\hline $\mathrm{C} 9-\mathrm{C} 10$ & 1.393 & 1.466 \\
\hline $\mathrm{C} 10-\mathrm{C} 11$ & 1.391 & 1.347 \\
\hline $\mathrm{C} 11-\mathrm{C} 12$ & 1.397 & 1.460 \\
\hline
\end{tabular}




\section{Continued}

\begin{tabular}{|c|c|c|}
\hline $\mathrm{C} 12-\mathrm{C} 13$ & 1.390 & 1.336 \\
\hline $\mathrm{C} 13-\mathrm{C} 8$ & 1.401 & 1.511 \\
\hline $\mathrm{C} 9-\mathrm{O} 2$ & 1.354 & 1.212 \\
\hline $\mathrm{O} 2-\mathrm{H} 13$ & 0.973 & - \\
\hline $\mathrm{C} 6-\mathrm{C} 1-\mathrm{C} 2$ & 118.12 & 119.75 \\
\hline $\mathrm{C} 1-\mathrm{C} 2-\mathrm{C} 3$ & 121.92 & 121.66 \\
\hline $\mathrm{C} 2-\mathrm{C} 3-\mathrm{C} 4$ & 120.16 & 119.26 \\
\hline C3-C4-C5 & 120.96 & 121.66 \\
\hline C4-C5-C6 & 119.47 & 119.05 \\
\hline $\mathrm{C} 5-\mathrm{C} 6-\mathrm{C} 1$ & 119.35 & 118.60 \\
\hline C5-C6-C14 & 117.26 & 117.92 \\
\hline C6-C14-C15 & 121.22 & 121.05 \\
\hline C14-C15-C16 & 121.17 & 120.86 \\
\hline $\mathrm{C} 15-\mathrm{C} 16-\mathrm{C} 17$ & 119.29 & 119.62 \\
\hline C16-C17-C5 & 120.98 & 120.99 \\
\hline C17-C5-C6 & 120.06 & 119.57 \\
\hline $\mathrm{C} 1-\mathrm{C} 2-\mathrm{O} 1$ & 118.57 & 121.68 \\
\hline $\mathrm{C} 3-\mathrm{C} 2-\mathrm{O} 1$ & 119.50 & 116.60 \\
\hline $\mathrm{C} 2-\mathrm{O} 1-\mathrm{H} 7$ & 109.29 & - \\
\hline $\mathrm{C} 2-\mathrm{C} 1-\mathrm{C} 7$ & 116.16 & 115.59 \\
\hline C6-C1-C7 & 125.73 & 124.62 \\
\hline $\mathrm{C} 1-\mathrm{C} 7-\mathrm{H} 8$ & 114.08 & 118.97 \\
\hline $\mathrm{C} 1-\mathrm{C} 7-\mathrm{N} 1$ & 125.64 & 125.25 \\
\hline $\mathrm{H} 8-\mathrm{C} 7-\mathrm{N} 1$ & 120.25 & 115.76 \\
\hline C7-N1-C8 & 121.27 & 118.22 \\
\hline $\mathrm{C} 2-\mathrm{O} 1-\mathrm{C} 8$ & - & 118.95 \\
\hline O1-C8-N1 & - & 116.49 \\
\hline $\mathrm{O} 1-\mathrm{C} 8-\mathrm{C} 9$ & - & 105.73 \\
\hline N1-C8-C9 & 114.43 & 107.54 \\
\hline N1-C8-C13 & 126.56 & 107.49 \\
\hline C8-C9-C10 & 120.37 & 117.39 \\
\hline C9-C10-C11 & 119.69 & 120.89 \\
\hline $\mathrm{C} 10-\mathrm{C} 11-\mathrm{C} 12$ & 120.61 & 122.62 \\
\hline $\mathrm{C} 11-\mathrm{C} 12-\mathrm{C} 13$ & 119.82 & 121.49 \\
\hline $\mathrm{C} 12-\mathrm{C} 13-\mathrm{C} 8$ & 120.56 & 121.90 \\
\hline $\mathrm{C} 13-\mathrm{C} 8-\mathrm{C} 9$ & 118.93 & 114.23 \\
\hline $\mathrm{C} 8-\mathrm{C} 9-\mathrm{O} 2$ & 119.75 & 119.08 \\
\hline $\mathrm{C} 10-\mathrm{C} 9-\mathrm{O} 2$ & 119.88 & 123.53 \\
\hline C9-O2-H13 & 105.52 & - \\
\hline
\end{tabular}


Table 5. Electronic energies for Schiff base neutral molecule and radicals in gas phase at B3LYP/6-311G(d,p) level of theory.

\begin{tabular}{cccccccc}
\hline S.No. & Salen & $\begin{array}{c}\text { Energy } \\
\text { (Hartree) }\end{array}$ & $\begin{array}{c}\text { Dipole } \\
\text { moment }\end{array}$ & $\begin{array}{c}\text { Electron } \\
\text { Affinity }\end{array}$ & $\begin{array}{c}\text { Ionization } \\
\text { Potential }\end{array}$ & Hardness & Electronegativity \\
\hline 1. & SA & -707.3695 & 1.7675 & 802.9496 & 798.4321 & -2.2586 & 800.6909 \\
2. & AA & -746.6926 & 2.8269 & 1273.5922 & 1269.0747 & -2.2586 & 1271.3335 \\
3. & VA & -821.6521 & 3.1964 & 235.31669 & 235.3167 & $0.05 \times \mathrm{x} \mathrm{e}^{-3}$ & 235.3167 \\
4. & NA & -861.0521 & 4.8823 & 1038.2778 & 1033.7603 & -2.2588 & 1036.0191 \\
\hline
\end{tabular}

Hardness, $\eta=\left[\frac{I P-E A}{2}\right]$.

Bond polarity is one of the factors that determine the physicochemical property of molecules. The calculated values of the total dipole moments, which signifies the relatively polarized nature of the systems and they are soluble in polar solvents like $\mathrm{CH}_{3} \mathrm{CN}, \mathrm{DMSO}, \mathrm{CHCl}_{3}$, etc.

The computed NMR chemical shifts for SA, AA, VA and NA at the DFT level of theory are in acceptable agreement with the experimental data. Differences between the calculated and measured values may be a result of solvent interactions.

The ${ }^{13} \mathrm{C}-\mathrm{NMR}$ chemical shifts of selected carbons were calculated on the optimized structures of SA, AA, VA and NA using GIAO/DFT method with B3LYP/6-311(d,p) basis set for all atoms. Calculated and measured ${ }^{13} \mathrm{C}$ chemical shifts of selected atoms are numbered in Figure 6.

\subsubsection{HOMO-LUMO Orbital Distribution}

The HOMO-LUMO energy (Figure 7 and Table 6) which characterizes the ability of electron-giving is appropriate to represent the free radical scavenging efficiency of phenolic compounds because the process to inhibit auto-oxidation may include the electron-transfer besides the abstraction of the $\mathrm{H}$-atom [12]. The HOMO $(-0.2070,-0.2040,-0.2160$ and -0.2040 Hartree $)$ and the LUMO $(-0.0590,-0.0440,-0.0750$ and -0.0790 Hartree $)$ for the compounds SA, AA, VA, NA respectively, are $\pi$-like orbitals confirmed by UV-Vis spectra and they are delocated in the whole molecule of all the salen compounds. Considering the disposition of HOMO and LUMO orbitals, the -OH group of 2 and 9-position is easily attacked by either the electrophilic or nucleophilic agents, such as radicals, metal ions and $\mathrm{O}_{2}$. Indeed, $\mathrm{HOMO}$ and LUMO orbitals for four compounds are localized on $\mathrm{A}$ and $\mathrm{B}$ ring, conjugated double bond nature in the phenyl and naphthyl moiety and on the A and B conjugated ring salen compounds [27] [28].

\subsubsection{Spin Density Distribution}

The spin density is often considered to be a more realistic parameter which provides a better representation of the reactivity [6] [9] of salen moiety. It should be pointed out that the more delocalized the spin density in the salen compound is, the easier the radical is formed [5]. In the Figure 8, the spin densities of all the radicals mainly distribute on the phenolic oxygen atom and the phenyl ring $\mathrm{A}$ 


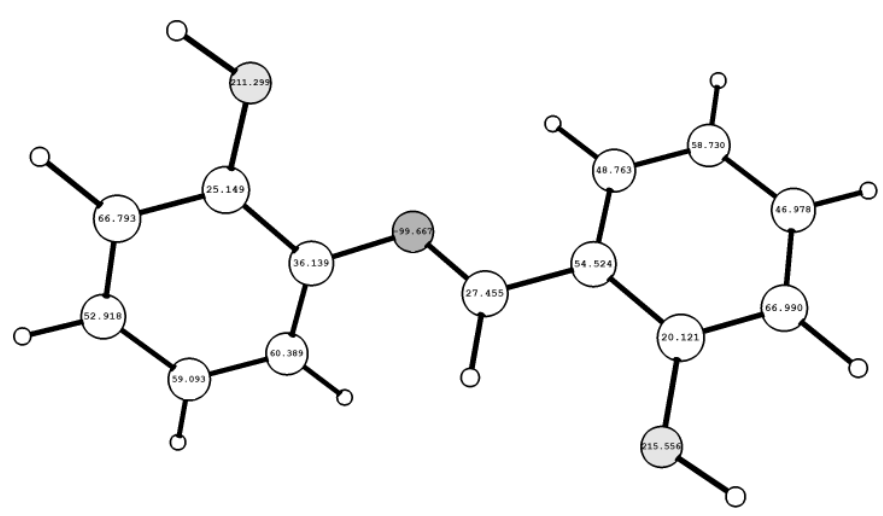

SA-NMR

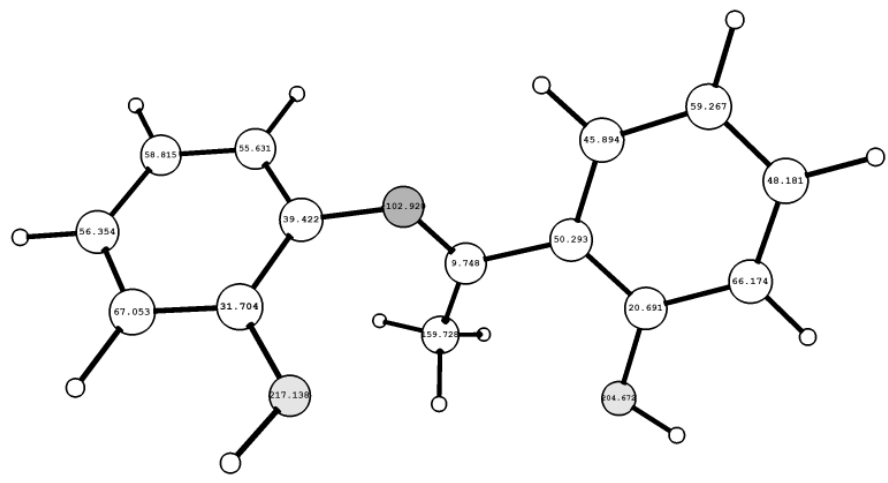

AA-NMR

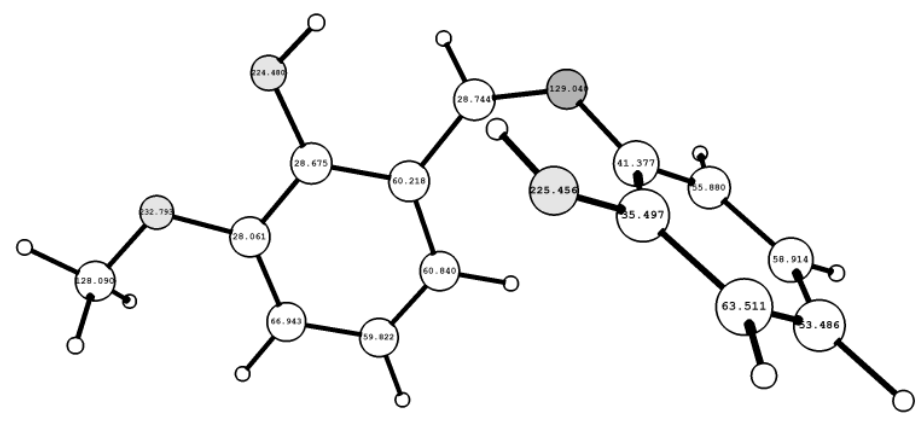

VA-NMR

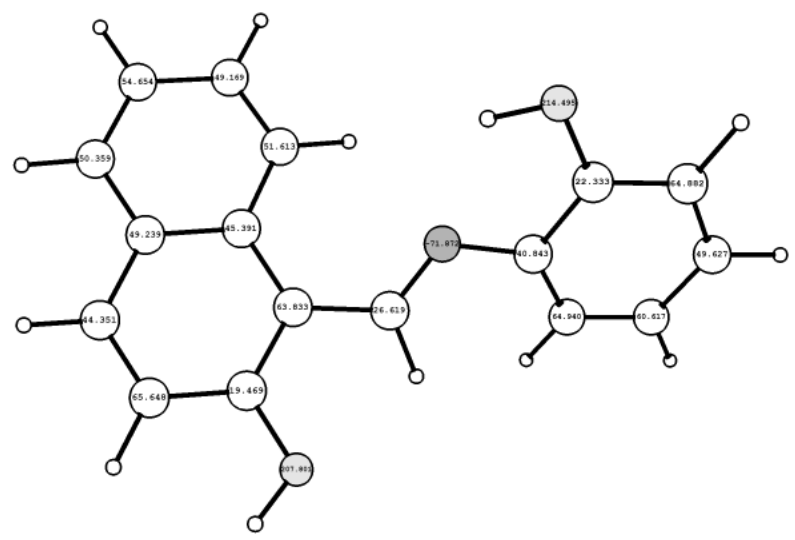

NA-NMR

Figure 6. NMR shielding of salen compounds. 


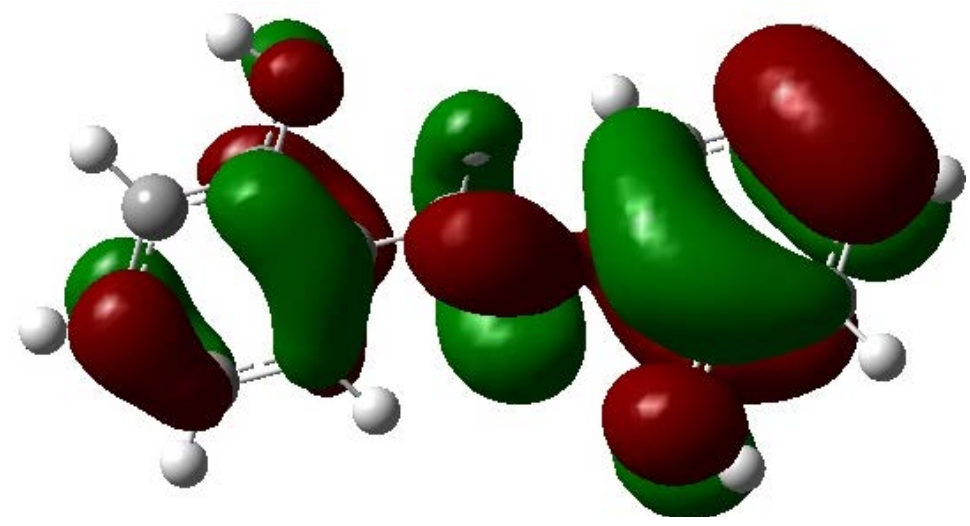

HOMO-Neutral SA

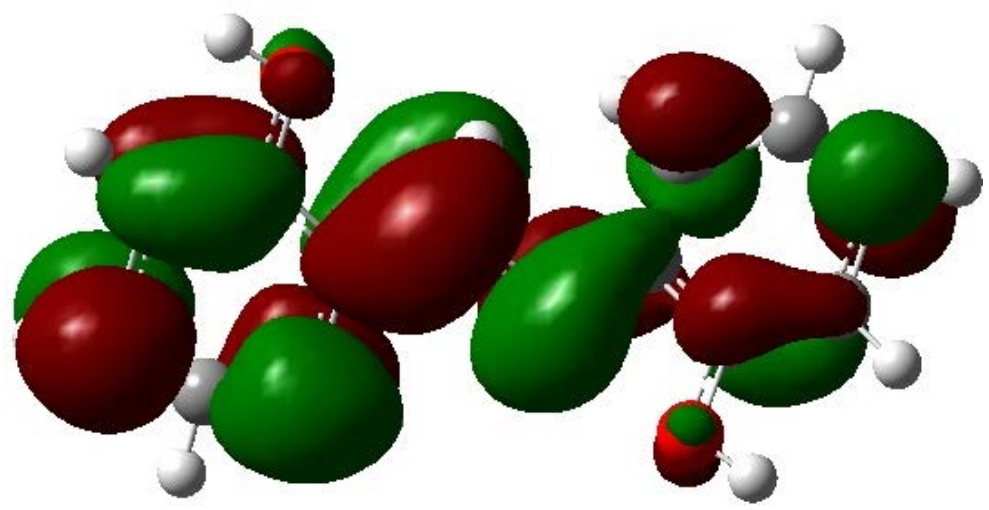

LUMO-Neutral SA

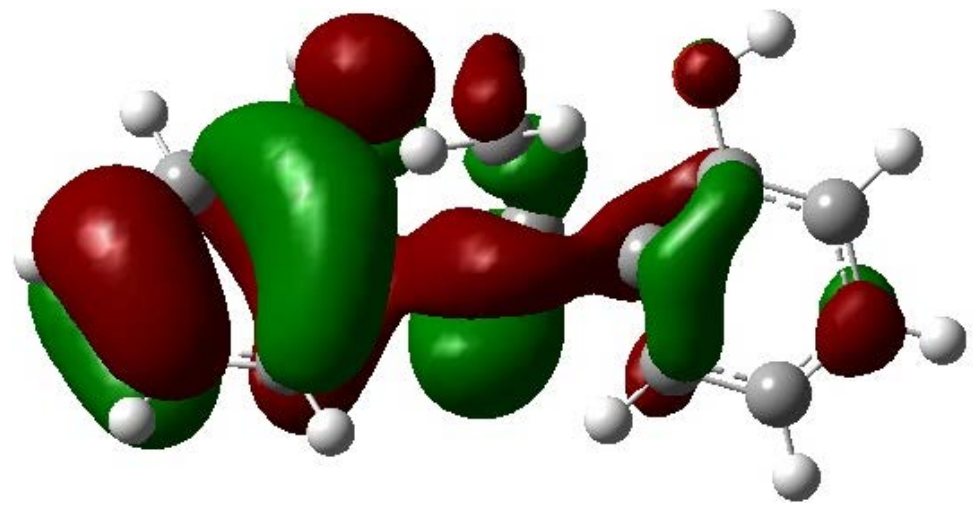

HOMO-Neutral AA

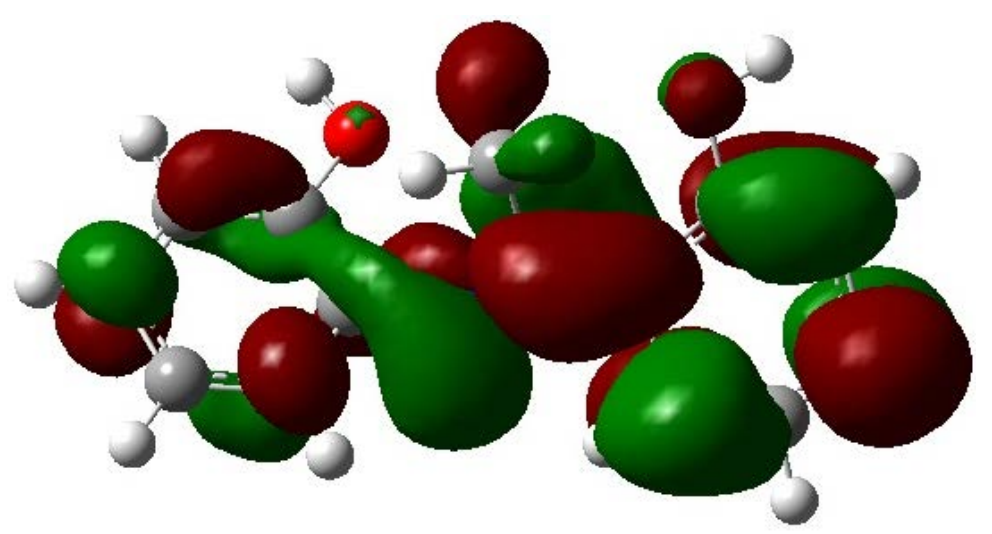

LUMO-Neutral AA 


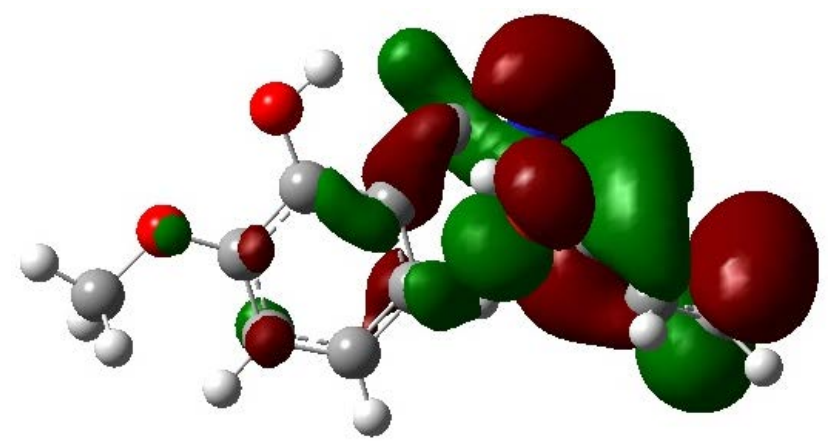

HOMO-Neutral VA

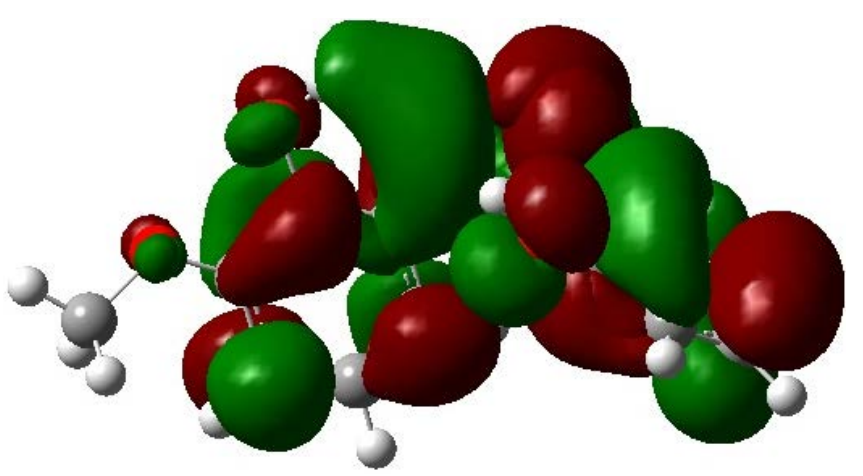

LUMO-Neutral SA

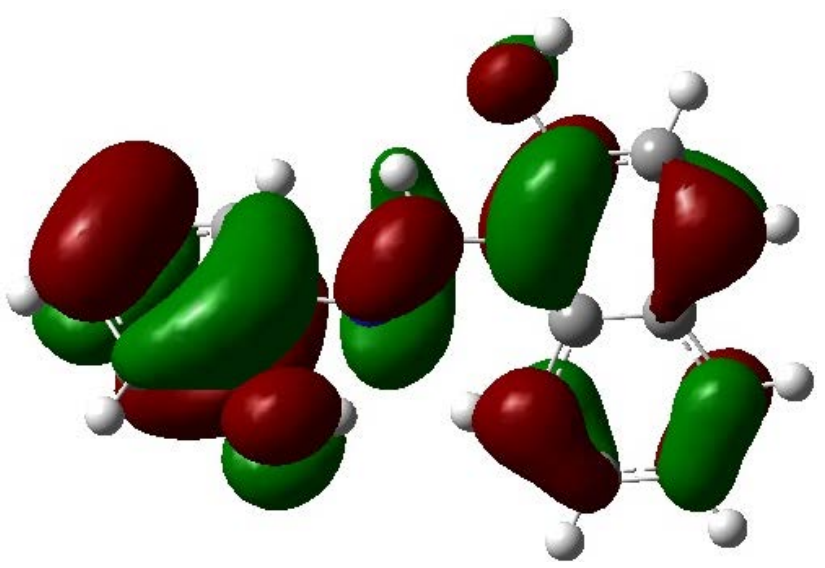

HOMO-NA

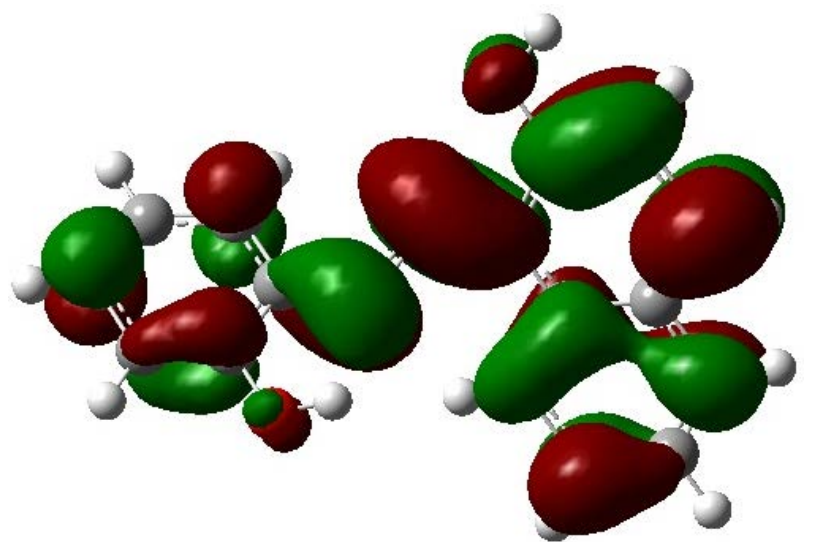

LUMO-Neutral NA

Figure 7. HOMO-LUMO diagrame of salen compounds. 


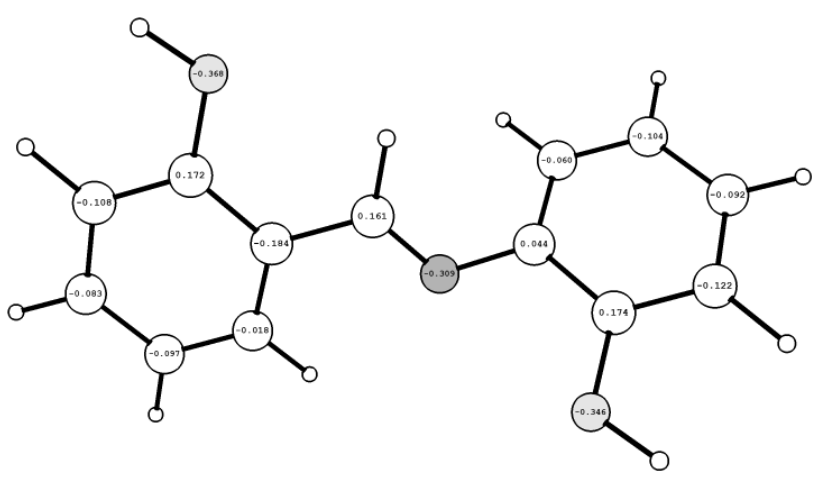

Spin Density-SA

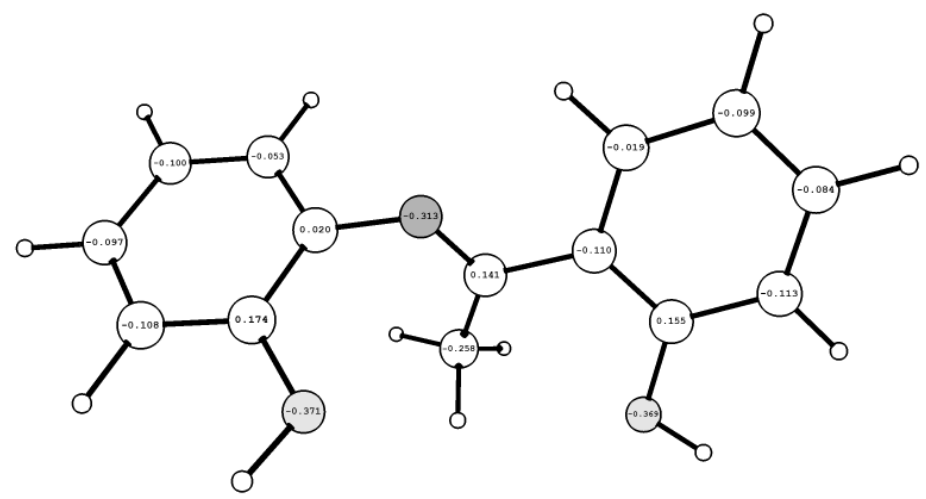

Spin Density-AA

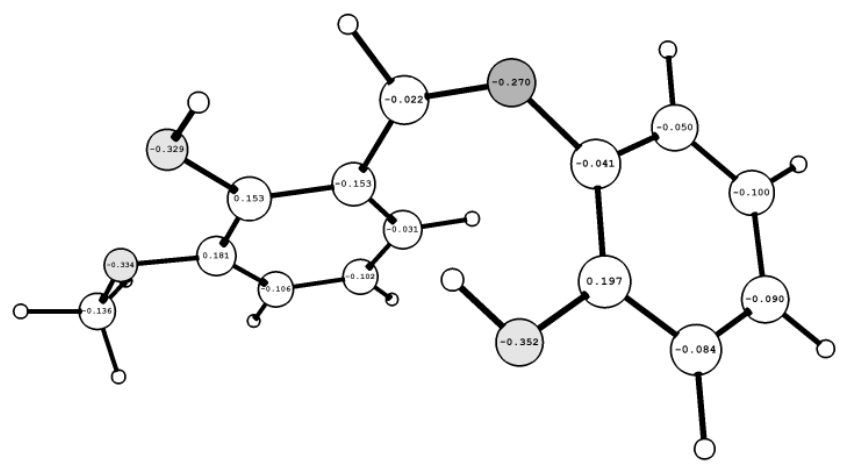

Spin Density-VA

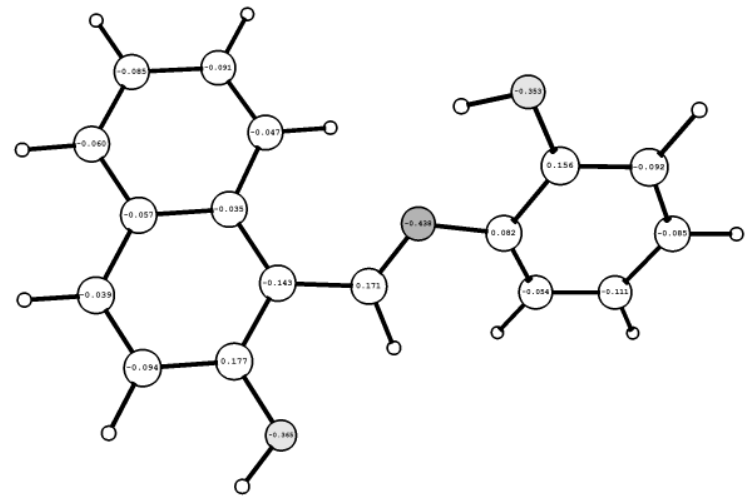

Spin Density-NA

Figure 8. Spin density distribution analaysis of salen compounds. 
Table 6. Total energies, Frontier orbital energies, Softness, $\mathrm{E}_{\text {gap }}$ and EI in gas phase calculated at B3LYP/6-311G(d,p) level of theory.

\begin{tabular}{ccccc}
\hline $\begin{array}{c}\text { Electronic } \\
\text { Parameter/molecule }\end{array}$ & SA & AA & VA & NA \\
\hline $\mathrm{E}_{\mathrm{T}}[$ Hartree $]$ & -707.3695 & -746.6926 & -821.6521 & -861.0521 \\
Dipole moment & 1.7675 & 2.8269 & 3.1964 & 4.8823 \\
$\mathrm{E}_{\text {HOMO }}[$ Hartree $]$ & -0.2070 & -0.2040 & -0.2160 & -0.2040 \\
$\mathrm{E}_{\text {LUMO }}[$ Hartree $]$ & -0.0590 & -0.0440 & -0.0750 & -0.0790 \\
$\Delta \mathrm{E}_{\text {gap }}[\mathrm{eV}]$ & 3.5506 & 4.3538 & 3.8368 & 3.4014 \\
Softness $[\mathrm{eV}]$ & 0.2214 & 0.2214 & - & 0.2214 \\
\hline
\end{tabular}

Table 7. All $\beta$ (a.u.) components and $\beta_{\text {(tot) }} \times 10^{-31}$ (esu) value calculated using DFT level of theory for salencompounds ( 1 a.u. $\left.=8.6393 \times 10^{-33} \mathrm{esu}\right)$.

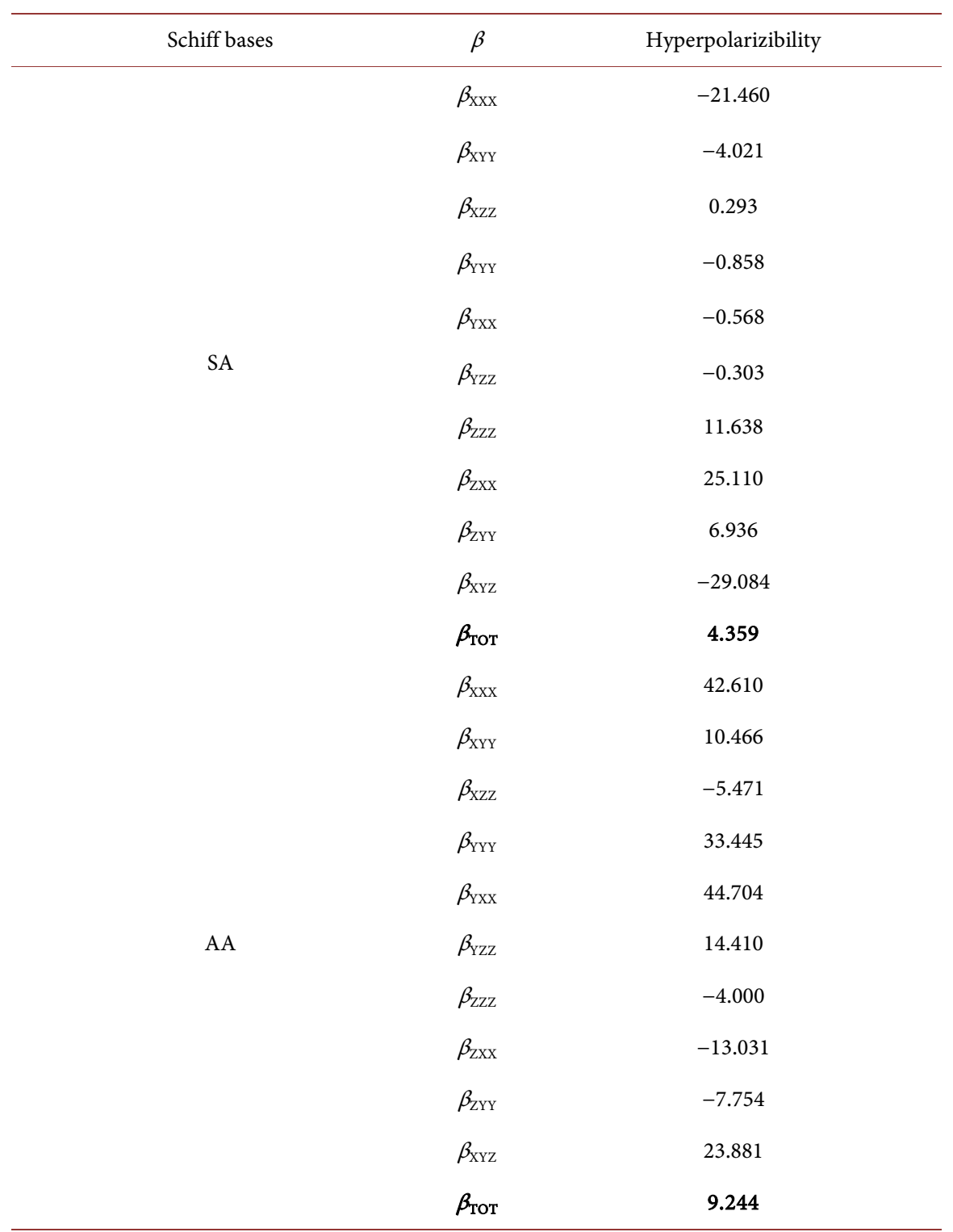




\section{Continued}

\begin{tabular}{|c|c|c|}
\hline & $\beta_{\mathrm{xxx}}$ & 101.759 \\
\hline & $\beta_{\mathrm{XYY}}$ & 1.365 \\
\hline & $\beta_{\mathrm{xzz}}$ & 1.976 \\
\hline & $\beta_{\mathrm{YYY}}$ & -38.170 \\
\hline & $\beta_{\mathrm{YXX}}$ & -62.018 \\
\hline \multirow[t]{11}{*}{ VA } & $\beta_{\mathrm{YZZ}}$ & -13.843 \\
\hline & $\beta_{\mathrm{ZZZ}}$ & 0.373 \\
\hline & $\beta_{\mathrm{ZXX}}$ & 5.855 \\
\hline & $\beta_{\mathrm{ZYY}}$ & 8.194 \\
\hline & $\beta_{\mathrm{XYZ}}$ & 30.912 \\
\hline & $\beta_{\mathrm{TOT}}$ & 13.455 \\
\hline & $\beta_{\mathrm{xxx}}$ & 28.769 \\
\hline & $\beta_{\mathrm{XYY}}$ & 51.498 \\
\hline & $\beta_{\mathrm{xzz}}$ & -5.941 \\
\hline & $\beta_{\mathrm{YYY}}$ & 54.654 \\
\hline & $\beta_{\mathrm{YXX}}$ & 24.052 \\
\hline \multirow[t]{6}{*}{ NA } & $\beta_{\mathrm{YZZ}}$ & 8.908 \\
\hline & $\beta_{\mathrm{ZZZ}}$ & 7.978 \\
\hline & $\beta_{\mathrm{ZXX}}$ & -0.190 \\
\hline & $\beta_{\mathrm{ZYY}}$ & -1.286 \\
\hline & $\beta_{\mathrm{XYZ}}$ & 13.656 \\
\hline & $\beta_{\mathrm{TOT}}$ & 10.003 \\
\hline
\end{tabular}

and $\mathrm{B}$. Comparing all the radicals, we find that the radicals have almost the same spin density distribution with each other, indicating the presence of additional hydroxyl or o,o-dihydroxyl group on A and B-ring has almost no influence on the spin density distribution [27] [28].

\subsubsection{Molecular Electrostatic Potential Surface (MEP) Study}

Electrostatic potential surfaces are mainly used to study the reactive species of electrophilic or nucleophilic attacks/substitution in the chemical reactions, biological process, catalysis and also molecular modeling. Electrostatic potential mapped surface displays the molecular size, shape and potential values. In this study, 3 dimensional surfaces of mapped onto the constant electron density surface is as shown in Figure 9. Different values of electrostatic potential at the surface are represented by different colors [29].

\subsubsection{Computed Non-Linear Optical (NLO) Properties}

The NLO response calculation was performed on the optimized geometry using the same level of theory. The first static hyperpolarizability is a third rank tensor that can be described by a $3 \times 3 \times 3$ matrix. The 27 components of the $3 \mathrm{D}$ matrix 


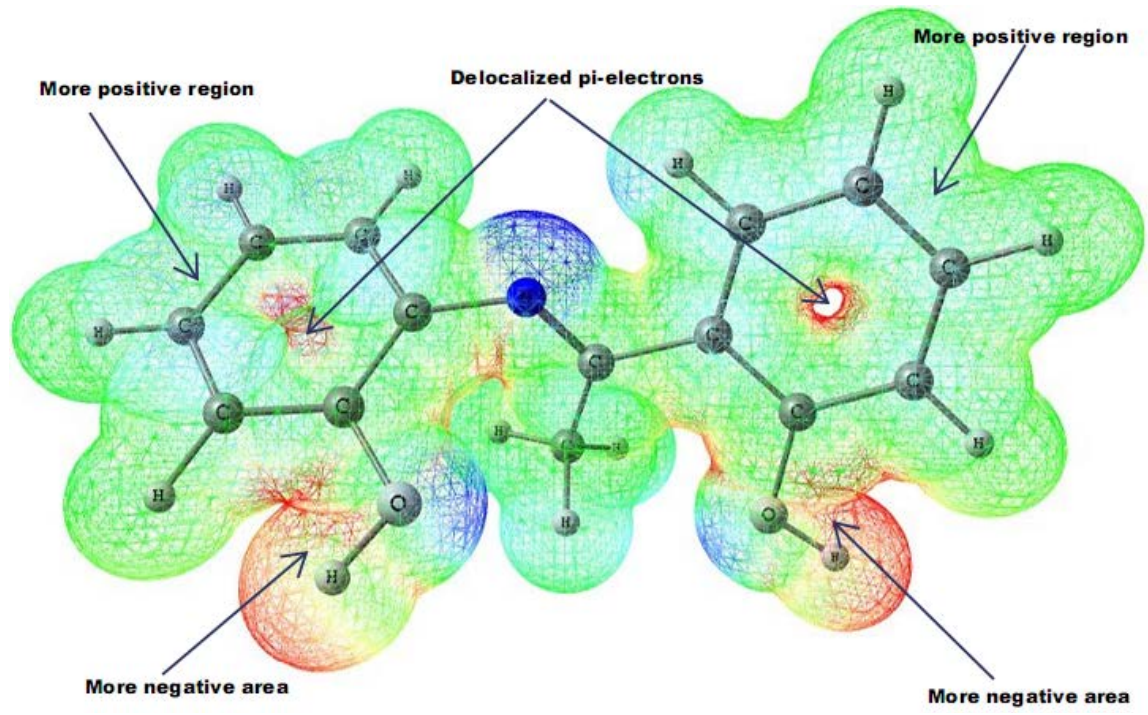

Figure 9. Molecular Electrostatic Potential surface diagram of AA.

can be reduced to 10 components due to the Kleinman symmetry [27] [30] ( $\beta_{x y y}$ $=\beta_{y x y}=\beta_{y y x} \beta_{y y z}=\beta_{y z y}=\beta_{z y y} \ldots$, likewise other permutations also take same value). It can be given in the lower tetrahedral format. The output from Gaussian $03 \mathrm{~W}$ provides 10 components of this matrix as $\beta_{x x p} \beta_{x x p} \beta_{x y y} \beta_{y y p} \beta_{x x z} \beta_{x y z} \beta_{y y z}$ $\beta_{x z z} \beta_{y z z}$ and $\beta_{z z z}$ respectively. Many types of hyperpolarizabilities have been discussed in the literature [31]. When reporting a single value of $\beta$, one of the common formats is to simply treat the three independent values for $\beta$ as a quasi-Pythagorean problem and solve for the average $\beta$ by Equation (5):

$$
\beta_{\text {tot }}=\left(\beta_{x}^{2}+\beta_{y}^{2}+\beta_{z}^{2}\right)^{1 / 2}
$$

The complete equation for calculating the magnitude of the total first statichyperpolarizability from Gaussian $03 \mathrm{~W}$ output is given as Equation (6):

$$
\beta_{t o t}=\left[\left(\beta_{x x x}+\beta_{x y y}+\beta_{x z z}\right)^{2}+\left(\beta_{y y y}+\beta_{y x x}+\beta_{y z z}\right)^{2}+\left(\beta_{z z z}+\beta_{z x x}+\beta_{z y y}\right)^{2}\right]^{1 / 2}
$$

Since these $\beta$ values of the first static hyperpolarizability $(\beta)$ tensors of the output file of Gaussian $03 \mathrm{~W}$ are reported in atomic units (a.u.), the calculated values were converted into electrostatic units $\left(1\right.$ a.u. $\left.=8.6393 \times 10^{-33} \mathrm{esu}\right)$. The first static hyperpolarizability $(\beta)$ values of these Schiff bases were calculated under static electronic field by the DFT (B3LYP/(6-311G(d,p)) method. From Table 7, compounds NA, VA and AA have larger the first static hyperpolarizability $\left(\beta_{t o t}\right)$ values than those of compound SA. As mentioned above, the DFT results show that the LUMO orbitals of all compounds were obtained from the linear combination of the orbitals of phenyl moiety, the compounds AA, VA and $\mathrm{NA}$ with an electron-donating and withdrawing group $\left(\mathrm{CH}_{3}, \mathrm{OCH}_{3}\right.$ and naphthyl) have a larger $\beta_{\text {tot }}$ value compare with compound SA. Hence the compound VA was predicted to have larger NLO property. The energy difference $\left(\Delta E_{g a p}\right)$ between the HOMO and the LUMO orbitals 3.5506, 4.3538, 3.8368, $3.4014 \mathrm{eV}$ for the compounds SA, AA, VA, NA respectively has a larger influence on the 
$\beta_{\text {tot }}$ value [32] [33]. To understand the relationship between the static hyperpolarizabilities and the HOMO-LUMO energy gap, viz. the compound VA (3.4014 $\mathrm{eV})$ lower the HOMO-LUMO energy gap show that the larger the $\beta_{\text {tot }}(13.455$ a.u) value.

\subsection{Conclusions}

Schiff bases (1-4) have been successfully synthesized and characterized by elemental analysis, FT-IR, UV-Vis, NMR spectroscopy and cyclic voltammetry. The evaluation of the quantum mechanical studies reveal significant activity in structure optimization, PES, vibrational study, NMR Chemical shifts, spin density, hardness, electronegativity, dipole moment, $E_{\mathrm{HOMO}} / E_{\mathrm{LUMO}}, \Delta E_{\text {gap }}$ and Softness which provide the evidence for a very strong positive correlation between experimental and theoretical predictions. These compounds also have towards considerable antioxidant activity along with potential to prevent DNA oxidative damage by free radicals.

Comparison between the four considered molecules indicates compound VA that requires the lowest energy for both $\mathrm{H}$ atom and electron transfer mechanisms. This theoretical approach confirms the important role of A and B ring in exhibiting antioxidant properties. Inspection of deprotonation processes of dihydroxyl groups have shown that, $\pi$ electron delocalization of phenyl ring A and B (evidenced from UV-Vis study) plays a major role in the stabilization of products and thus in the lowering of the associated energies. The variables related to the chemical potential allow classifying salen type of Schiff base compounds that has the tendency to give electrons more than to attract them, which demonstrates biological importance.

In addition to that in order to understand the relationship between the $\beta_{t o t}$ values and the substitution groups of salen compounds, the frontier orbital compositions have been analyzed and the energy gaps between the HOMO and LUMO orbitals were also calculated. The compound VA with electron-donating group $\left(-\mathrm{OCH}_{3}\right)$ will produce the larger $\beta_{\text {tot }}$ value (13.455 a.u) than lower $\beta_{\text {tot }}$ $(10.003,9.244,4.359$ a.u $)$ for the compounds NA, AA, SA respectively. The energy gaps between the HOMO and LUMO orbitals show that the lower the HOMO-LUMO energy gap, larger the first static hyperpolarizability $\left(\beta_{t o t}\right)$.

The findings of this work sustenance the view that some of these synthesized compounds are promising sources of potential drugs that may be efficient as preventive agent(s) in some diseases.

\section{References}

[1] Ayman, A., Aziz, A., Salem, M.A.N., Sayed, M.A. and Aboaly, M.M. (2012) Synthesis, Structural Characterization, Thermal Studies, Catalytic Efficiency and Antimicrobial Activity of Some M(II) Complexes with ONO TRIDENTATE SCHIFF BASE N-Salicylidene-o-Aminophenol (saphH2). Journal of Molecular Structure, 1010, 130-138.

[2] Opstal, T. and Verpoort, F. (2003) Synthesis of Highly Active Ruthenium Indenylidene Complexes for Atom-Transfer Radical Polymerization and Ring-Opening- 
Metathesis Polymerization. Angewandte Chemie International Edition, 42, 28762886. https://doi.org/10.1002/anie.200250840

[3] da Silva, C.M., da Silva, D.L., Modolo, L.V., Alves, R.B., Martins, C.V.B., de Resende, M.A. and de Fátima, A. (2011) Schiff Bases: A Short Review of Their Antimicrobial Activities. Journal of Advanced Research, 2, 1-8.

[4] Sztanke, K., Maziarka, A., Osinka, A. and Sztanke, M. (2013) An Insight into Synthetic Schiff Bases Revealing Antiproliferative Activities in Vitro. Bioorganic \& Me dicinal Chemistry, 21, 3648-3666.

[5] Hameed, A., Al-Rashida, M., Uroos, M., Ali, S.A. and Khan, K.M. (2017) Schiff Bases in Medicinal Chemistry: A Patent Review (2010-2015). Expert Opinion on Therapeutic Patents, 27, 63-79. https://doi.org/10.1080/13543776.2017.1252752

[6] Sirajuddin, M., Uddin, N., Ali, S. and Tahir, M.N. (2013) Potential Bioactive Schiff Base Compounds: Synthesis, Characterization, X-Ray Structures, Biological Screenings and Interaction with Salmon Sperm DNA. Spectrochimica Acta Part A: Molecular and Biomolecular Spectroscopy, 116, 111-121.

[7] Govindarasu, K. and Kavitha, E. (2014) Vibrational Spectra, Molecular Structure, NBO, NMR, UV, First Order Hyperpolarizability, Analysis of (S)-(-)-N-(5-Nitro-2Pyridyl) Alaninol by Density Functional Theory. Spectrochimica Acta Part A: Molecular and Biomolecular Spectroscopy, 127, 498-510.

[8] Chandra, S. and Saneetika, X. (2004) EPR, Magnetic and Spectral Studies of Copper(II) and Nickel(II) Complexes of Schiff Base Macrocyclic Ligand Derived from Thiosemicarbazide and Glyoxal. Spectrochimica Acta Part A: Molecular and Biomolecular Spectroscopy, 60, 147-153.

[9] Finkel, T. and Holbrook, N.J. (2000) Review Article Oxidants, Oxidative Stress and the Biology of Ageing. Nature, 408, 239-247. https://doi.org/10.1038/35041687

[10] Zhou, L., Cai, P., Feng, Y., Cheng, J., Xiang, H., Liu, J., Wu, D. and Zhou, X. (2012) Synthesis and Photophysical Properties of Water-Soluble Sulfonato-Salen-Type Schiff Bases and Their Applications of Fluorescence Sensors for $\mathrm{Cu}^{2+}$ in Water and Living Cells. Analytica Chimica Acta, 735, 96-106.

[11] Priyarega, S., Prabhakaran, R., Aranganayagam, K.R., Karvembu, R. and Natarajan, K. (2007) Synthetic and Catalytic Investigations of Ruthenium(III) Complexes with Triphenylphosphine/Triphenylarsine and Tridentate Schiff Base. Applied Organometallic Chemistry, 21, 788-793. https://doi.org/10.1002/aoc.1281

[12] Mazzi, U., Refosco, F., Tisato, F., Bandoli, G. and Nicolini, M. (1986) Mixed Triand Bi-Dentate Schiff-Base Complexes of Technetium(V) and Rhenium(V). The Crystal Structure of N-(2-Oxidophenyl)Salicylideneiminato-NOO')Oxo(8-Quinolinolato-NO)Technetium(V). Journal of the Chemical Society, Dalton Transactions, No. 8, 1623-1628. https://doi.org/10.1039/DT9860001623

[13] Tisato, F., Refosco, F., Mazzi, U., Bandolin, U. and Nicolini, M. (1987) Technetium(V) and Rhenium(V) Complexes with N-(2-Mercaptophenyl)Salicylideneiminate. Crystal Structure of Chloro(oxo)[N-(2-Sulphidophenyl)SalicylideneiminatoNOS]Technetium(V). Journal of the Chemical Society, Dalton Transactions, No. 7, 1693-1699. https://doi.org/10.1039/dt9870001693

[14] Priyarega, S., Tamizh, M.M., Karvembu, R., Prabhakaran, R. and Natarajan, K. (2011) Synthesis, Spectroscopic Characterization and Catalytic Oxidation Properties of ONO/ONS Donor Schiff Base Ruthenium(III) Complexes Containing $\mathrm{PPh}_{3} /$ $\mathrm{AsPh}_{3}$. Journal of Chemical Sciences, 123, 319-325. https://doi.org/10.1007/s12039-011-0087-2

[15] Frisch, M.J., Trucks, G.W., Schlegel, H.B., Scuseria, G.E., Robb, M.A., Cheeseman, J.R., Zakrzewski, V.G., Montgomery Jr., J.A., Stratmann, R.E., Burant, J.C., Dap- 
prich, S., Millam, J.M., Daniels, A.D., Kudin, K.N., Strain, M.C., Farkas, O., Tomasi, J., Barone, V., Cossi, M., Cammi, R., Mennucci, B., Pomelli, C., Adamo, C., Clifford, S., Ochterski, J., Petersson, G.A, Ayala, P.Y., Cui, Q., Morokuma, K., Malick, D.K., Rabuck, A.D., Raghavachari, K., Foresman, J.B., Cioslowski, J., Ortiz, J.V., Baboul, A.G., Stefanov, B.B., Liu, G., Liashenko, A., Piskorz, P., Komaromi, I., Gomperts, R., Martin, R.L., Fox, D.J., Keith, T., Al-Laham, M., Peng, C.Y., Nanayakkara, A., Gonzalez, C., Challacombe, M., Gill, P.M.W., Johnson, B., Chen, W., Wong, M.W., Andres, J.L., Gonzalez, C., Head-Gordon, M., Reploge, E.S. and Pople, J.A. (2005) Gaussian 03, Revision D.01. Gaussian, Inc., Pittsburgh, PA.

[16] Kohn, W. (1999) Nobel Lecture: Electronic Structure of Matter-Wave Functions and Density Functionals. Reviews of Modern Physics, 71, 1253.

https://doi.org/10.1103/RevModPhys.71.1253

[17] Becke, A.D. (1998) Density-Functional Exchange-Energy Approximation with Correct Asymptotic Behavior. Physical Review A, 38, 3098.

https://doi.org/10.1103/PhysRevA.38.3098

[18] Lee, C., Yang, W. and Parr, R.G. (1988) Development of the Colle-Salvetti Correlation-Energy Formula into a Functional of the Electron Density. Physical Review B, 37, 785. https://doi.org/10.1103/PhysRevB.37.785

[19] Frisch, M.J., Pople, J.A. and Binkley, J.S. (1984) Self-Consistent Molecular Orbital Methods 25. Supplementary Functions for Gaussian Basis Sets. The Journal of Chemical Physics, 80, 3265. https://doi.org/10.1063/1.447079

[20] Hehre, W.J., Radom, L., Schleyer, P.V.R. and Pople, J.A. (1986) Ab Initio Molecular Orbital Theory. Wiley, New York.

[21] Kohn, W., Becke, A.D. and Parr, R.G. (1996) Density Functional Theory of Electronic Structure. The Journal of Physical Chemistry, 100, 12974-12980. https://doi.org/10.1021/jp9606691

[22] Parr, R.G. and Pearson, R.G. (1983) Absolute Hardness: Companion Parameter to Absolute Electronegativity. Journal of the American Chemical Society, 105, 75127516. https://doi.org/10.1021/ja00364a005

[23] Parr, R.G., Szentpaly, L.V. and Liu, S. (1999) Electrophilicity Index. Journal of the American Chemical Society, 121, 1922-1924. https://doi.org/10.1021/ja983494x

[24] Chattaraj, P.K., Lee, H. and Parr, R.G. (1991) HSAB Principle. Journal of the American Chemical Society, 113, 1855-1856. https://doi.org/10.1021/ja00005a073

[25] Sadasivam, K. and Kumaresan, R. (2011) Antioxidant Behavior of Mearnsetin and Myricetin Flavonoid Compounds-A DFT Study. Spectrochimica Acta Part A: Molecular and Biomolecular Spectroscopy, 79, 282-293.

[26] Gabr, A.A. (1990) Spectrophotometric Studies on Some Schiff Bases deriveD from Benzidine. Spectrochimica Acta Part A: Molecular and Biomolecular Spectroscopy, 46, 1751-1757.

[27] Wright, J.S., Johnson, E.R. and Dilabio, G.A (2001) Predicting the Activity of Phenolic Antioxidants: Theoretical Method, Analysis of Substituent Effects, and Application to Major Families of Antioxidants. Journal of the American Chemical Society, 123, 1173-1183. https://doi.org/10.1021/ja002455u

[28] Sadasivam, K. and Kumaresan, R. (2011) Theoretical Investigation on the Antioxidant Behavior of Chrysoeriol and Hispidulin Flavonoid Compounds-A DFT Study. Computational and Theoretical Chemistry, 963, 227-235.

[29] Politzer, P. and Truhlar, D.G., Eds. (1981) Plenum, New York.

[30] Kleinman, D.A. (1962) Nonlinear Dielectric Polarization in Optical Media. Physical Review, 126, 1977. https://doi.org/10.1103/PhysRev.126.1977 
[31] Pipek, J. and Mezey, P.Z. (1989) A Fast Intrinsic Localization Procedure Applicable for $a b$ Initio and Semiempirical Linear Combination of Atomic Orbital Wave Functions. The Journal of Chemical Physics, 90, 4916. https://doi.org/10.1063/1.456588

[32] Wang, P., Zhu, P., Wang, C. and Ye, C. (1999) Theoretical Investigation and Molecular Design of Pyrazine Derivatives with Large Hyperpolarizabilities $(\beta)$. Journal of Molecular Structure. THEOCHEM, 459, 155-162.

[33] Tang, G.D., Jiang, Z.J., Li, R.Q., Zhang, J.F., Zhang, Y. and Zhang, C. (2009) DFT Studies on Nonlinear Optical Properties of Neutral Nest-Shaped Heterothiometallic [MOS3Py5Cu3X] ( $\mathrm{M}=\mathrm{Mo}, \mathrm{W} ; \mathrm{X}=\mathrm{F}, \mathrm{Cl}, \mathrm{Br}, \mathrm{I})$ Clusters. Spectrochimica Acta Part A: Molecular and Biomolecular Spectroscopy, 74, 228-232.

Submit or recommend next manuscript to SCIRP and we will provide best service for you:

Accepting pre-submission inquiries through Email, Facebook, LinkedIn, Twitter, etc. A wide selection of journals (inclusive of 9 subjects, more than 200 journals) Providing 24-hour high-quality service User-friendly online submission system Fair and swift peer-review system Efficient typesetting and proofreading procedure Display of the result of downloads and visits, as well as the number of cited articles Maximum dissemination of your research work

Submit your manuscript at: http://papersubmission.scirp.org/ Or contact ijoc@scirp.org 